\title{
Acoustic Nondestructive Testing and Measurement of Tension for Steel Reinforcing Members
}

Michael K. Mclnerney, Matthew W. Brenner, Sean W.

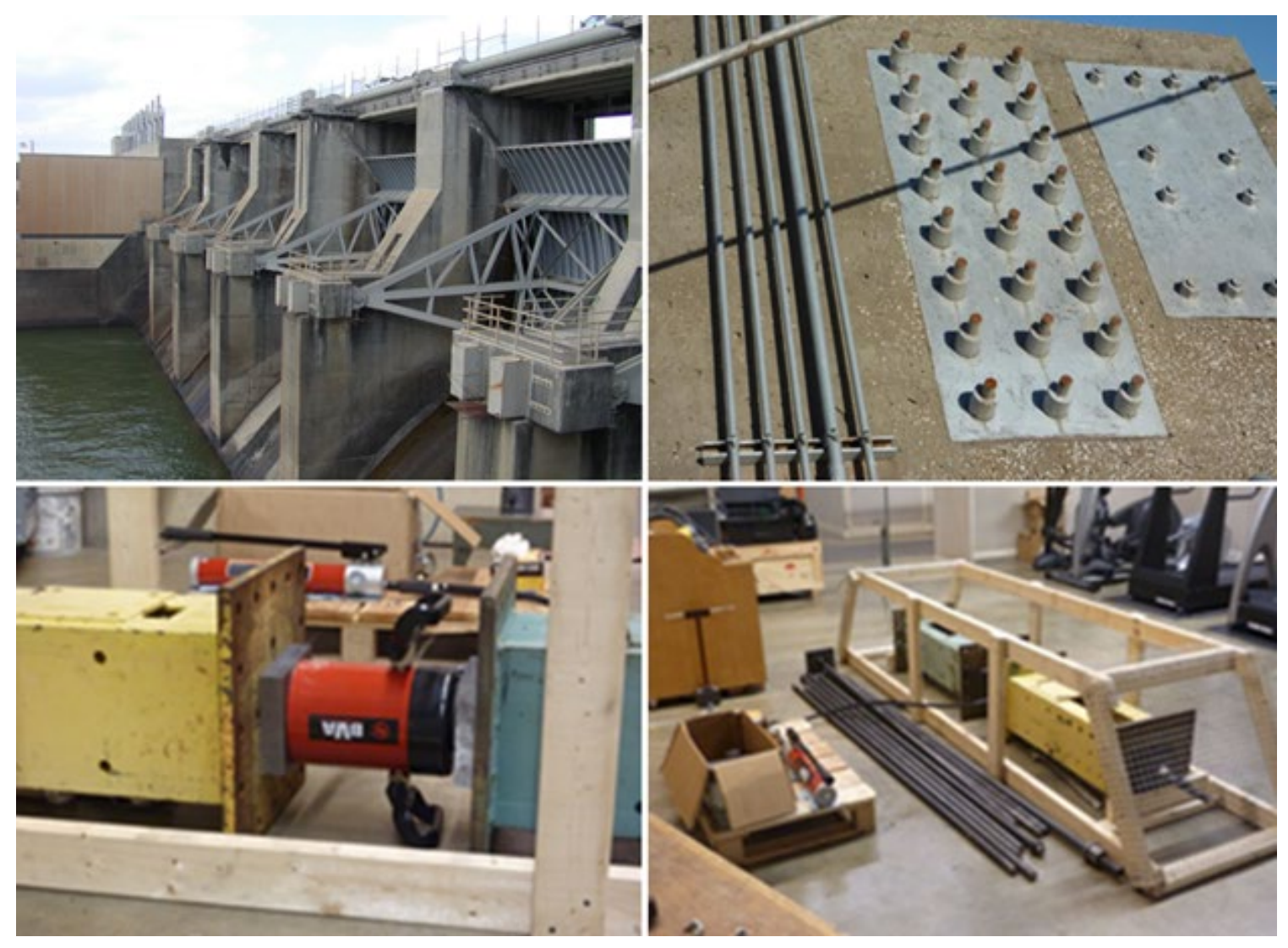


The U.S. Army Engineer Research and Development Center (ERDC) solves the nation's toughest engineering and environmental challenges. ERDC develops innovative solutions in civil and military engineering, geospatial sciences, water resources, and environmental sciences for the Army, the Department of Defense, civilian agencies, and our nation's public good. Find out more at www.erdc.usace.army.mil.

To search for other technical reports published by ERDC, visit the ERDC online library at http://acwc.sdp.sirsi.net/client/default. 


\section{Acoustic Nondestructive Testing and Measurement of Tension for Steel Reinforcing Members}

Michael K. Mclnerney, Matthew W. Brenner, Sean W. Morefield, and Robert A. Weber

U.S. Army Engineer Research and Development Center (ERDC) Construction Engineering Research Laboratory (CERL) 2902 Newmark Dr. Champaign, IL 61824

John M. Carlyle Carlyle Consulting 1009 Buckingham Way Yardley, PA 19067

Technical Report

Approved for public release; distribution unlimited.

Prepared for Headquarters, U.S. Army Corps of Engineers

Washington, DC 20314-1000

Under Navigation Systems Research Program, Project Number 611102AH68, Work Unit 98KJ26 


\section{Abstract}

Many concrete structures contain internal post-tensioned steel structural members that are subject to fracturing and corrosion. The major problem with conventional tension measurement techniques is that they use indirect and non-quantitative methods to determine whether there has been a loss of tension. This work developed an acoustics-based technology and method for making quantitative tension measurements of an embedded, tensioned steel member. The theory and model were verified in the laboratory using a variety of steel rods as test specimens. Field tests of the method were conducted at three Corps of Engineers dams. Measurements of the longitudinal and shear velocity were done on rods up to $50 \mathrm{ft} \mathrm{long.}$ Not all rods of this length were able to be measured and the quality and consistency of the signal varied. There were fewer problems measuring the longitudinal velocity than shear velocity. While the tension predictions worked in the laboratory tests, the tension could not be accurately calculated for any of the field sites because researchers could not obtain the longitudinal or shear velocities in an unstressed state, or precise measurements of the longitudinal and shear velocities due to the unknown precise length of the rods in the tensioned state.

DISCLAIMER: The contents of this report are not to be used for advertising, publication, or promotional purposes. Citation of trade names does not constitute an official endorsement or approval of the use of such commercial products. All product names and trademarks cited are the property of their respective owners. The findings of this report are not to be construed as an official Department of the Army position unless so designated by other authorized documents.

DESTROY THIS REPORT WHEN NO LONGER NEEDED. DO NOT RETURN IT TO THE ORIGINATOR. 


\section{Contents}

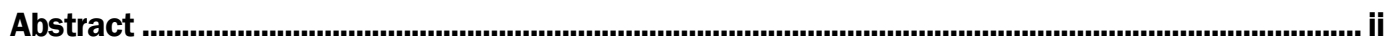

Figures and Tables...............................................................................................................................

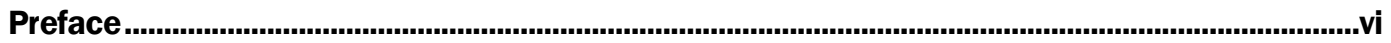

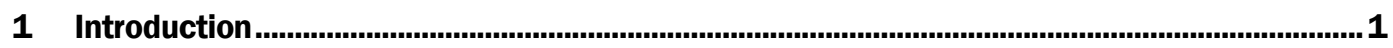

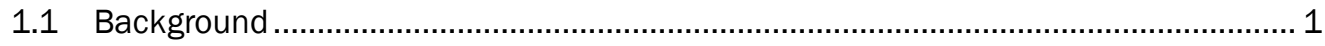

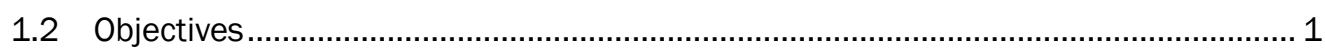

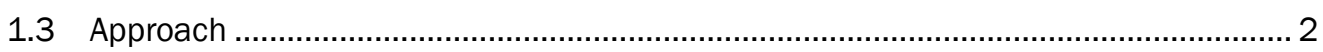

2 Tension Measurement Theory

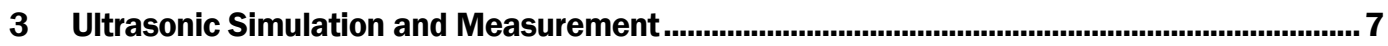

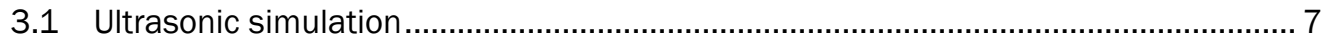

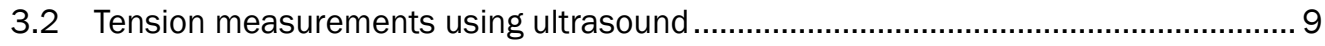

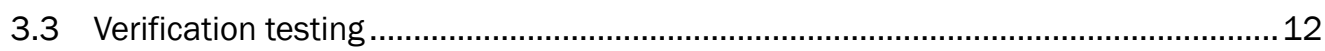

3.4 Corrosion Prediction using Ultrasound ............................................................. 16

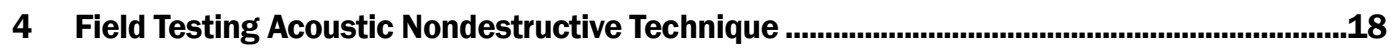

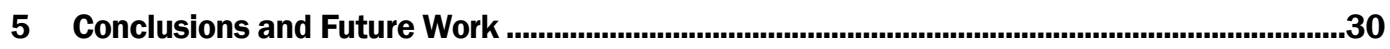

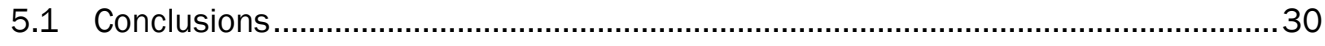

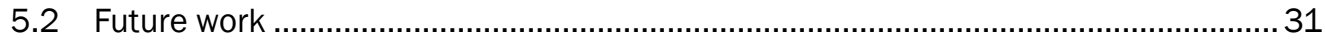

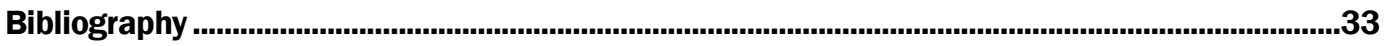

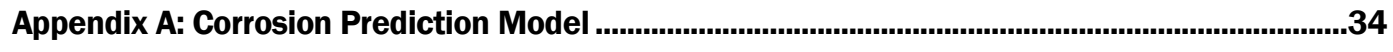

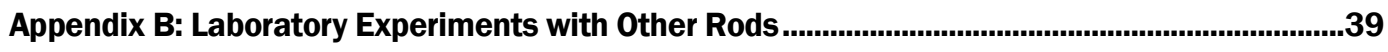

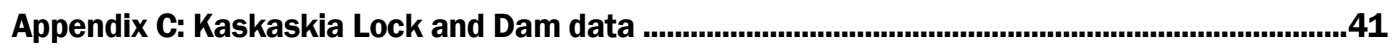

Report Documentation Page (SF 298) ........................................................................... 


\section{Figures and Tables}

\section{Figures}

1 Illustration of acoustic wave propagation

2 Ultrasonic instrument showing received signal from transducer mounted on left end of rod (top); simulation of signal propagation within the rod (bottom).................. 8

3 Simulation of echoes in sample rod (top) and actual screen shot from ultrasonic instrument display (bottom)

$4 \quad$ Model of $19 \times 1.25 \mathrm{in}$. (diameter) steel rod showing how first echo (green rays) and second echo (magenta) form (top); model showing how the first echo (green rays) and third echo (gray rays) form (bottom).

5 Pictures of the experimental setup for measuring tension and longitudinal and shear velocities

6 Pictures of the (upper left) transducers, (lower left) digital voltmeter used to readout the strain from the strain gauge, and (right) the flaw detector used in the experiments

$7 \quad$ Plots of the measured longitudinal and shear velocities as a function of applied stress for three independent trial runs of steel 1018 using a $2.25 \mathrm{MHz}$ transducer. The dashed line in (a) is the theoretically derived fitting function

8 Plots of the scaled longitudinal and shear velocities as a function of strain for independent trial runs of steel 1018 using a $2.25 \mathrm{MHz}$ transducer. Each set of data is fit to a linear curve and the average slope, given by $<m>$ is recorded.

9 Typical tainter gates and trunnions ……….......................................................................19

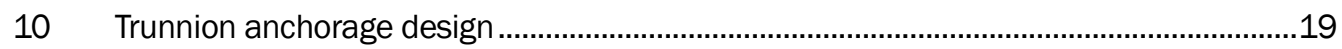

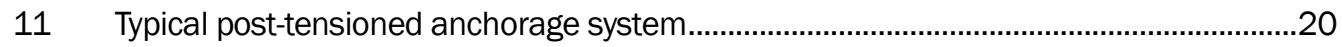

12 Failed anchor rod penetrating cover, showing one dimple in each cover indicating failed rods that did not penetrate (left); cover removed exposing anchor rods prior to testing (right)

13 Site of third field test, Kaskaskia Lock and Dam, Illinois ..................................................2

14 Rod ends at Kaskaskia Lock and Dam (left); broken rod (right) ......................................24

15 "Normal" rod with $2.25 \mathrm{MHz}$ sensor (left); "abnormal” rod with $2.25 \mathrm{MHz}$ sensor (right)

16 "Normal" rod with $2.25 \mathrm{MHz}$ sensor (left); "normal" rod with $1.0 \mathrm{MHz}$ sensor (right) 


\section{Tables}

1 Load-induced length change in a $19 \times 1.25$ in. (diameter) steel rod..............................10

2 Longitudinal and shear velocity in Block E West ..........................................................22

3 Longitudinal and shear velocity in Block E East ..................................................................23 


\section{Preface}

This study was conducted for Headquarters, US Army Corps of Engineers (HQUSACE) under the Navigation Systems Research Program, Work Unit 98KJ 26, Project Number 611102AH68, "High Power Acoustic NDT." The Program Manager for the Navigation Systems Research Program was Charles E. Wiggins, Coastal and Hydraulics Laboratory (CHL), U.S. Army Engineer Research and Development Center (ERDC). J eff Lillycrop was the Technical Director for Navigation, CHL.

The work was performed by the Materials and Structures (CFM) Branch of the Facilities Division (CF), Construction Engineering Research Laboratory (CERL). At the time of the preparation of this report, Vicki Vanblaricum was Chief, CFM and Donald Hicks was Chief, CF. The associated Technical Director was Kurt Kinnevan. The Deputy Director of CERL was Dr. Kirankumar V. Topudurti, and the Director was Dr. Lance D. Hansen.

The following personnel who supported this effort and are gratefully acknowledged for their assistance: George Poiroux (ret.) - South Atlantic Division, Mobile District (SAM-EN-DE); J effrey Stamper - Mississippi Valley Division, St. Louis District (CEMVS-EC-DA); J ames Hill (ret.) Mississippi Valley Division, St. Louis District, Kaskaskia River Project (CEMVS-OD-AK).

COL Ivan P. Beckman was Commander of ERDC, and Dr. David W. Pittman was the Director. 


\section{Introduction}

\subsection{Background}

Many concrete structures contain internal post-tensioned steel structural members that are subject to fracturing and corrosion. In large concrete structures such as locks and dams, corrosion of these steel components can lead to loss of tension and consequent severe problems, such as cracking of the concrete or fracturing of the steel. Although conventional tension measurement techniques exist to test internal post-tensioned steel structural members for loss of tension, the major problem with these techniques is that they use indirect and non-quantitative methods to determine whether there has been a loss of tension.

This work developed an acoustics-based technology and method for making quantitative tension measurements of an embedded, tensioned steel member. Acoustic waves are uniquely suited to this analytical task. They are nondestructive, they can travel long distances in engineered structures, and they can thoroughly interrogate a structure's integrity. Acoustic wave analysis can serve two critical purpose: they can determine bulk material properties, such as tension; and they can detect small defects, such as fractures and pores. An additional benefit is that acoustic measurements can be performed very quickly, usually in real time, although post-processing may be required.

\subsection{Objectives}

The objective of this work was to

1. Investigate the theoretical basis for bulk tension measurements

2. Develop an acoustic propagation model that will support the development of a nondestructive evaluation (NDE) technology that can quantitatively measure tension in structural steel reinforcing members

3. Test that theory and model in the laboratory using simple steel rods as test specimens

4. Investigate a technology application that addresses the problem of determining tension in concrete-embedded and post-tensioned reinforcement rods used in large Civil Works hydraulic structures. 


\subsection{Approach}

The objectives of this work were accomplished in the following steps:

1. A theoretical model was developed to relate the stress in a post-tensioned rod to a function of longitudinal and shear acoustic velocity.

2. The prediction of the theory was tested in a laboratory setting by placing rods in tension while the longitudinal and shear acoustic velocities were measured.

3. The acoustic velocities of post-tensioned rods were measured at dams in the field and an analysis was performed.

4. Results were analyzed, conclusions formulated, and recommendations for further work proposed. 


\section{Tension Measurement Theory}

A material's ultrasonic properties are fundamental to the understanding of wave behavior. There is much literature describing the theory and applications of ultrasonic waves (Auld 1990, Ensminger 1973, Filipczynski et al. 1966, and Krautkramer and Krautkramer 1990). Ultrasonic waves can propagate as both longitudinal and shear waves. Figure 1 shows the two different propagation modes. For longitudinal waves, the direction of particle motion is the same as the direction of propagation. For shear waves, the direction of particle motion is perpendicular to the direction of propagation. Longitudinal waves can exist in all media; shear waves exist only in solids. In steel, shear waves move about half the speed of longitudinal waves. Also, the shear wave is 4 to 10 times more attenuated than the longitudinal wave.

Figure 1. Illustration of acoustic wave propagation.

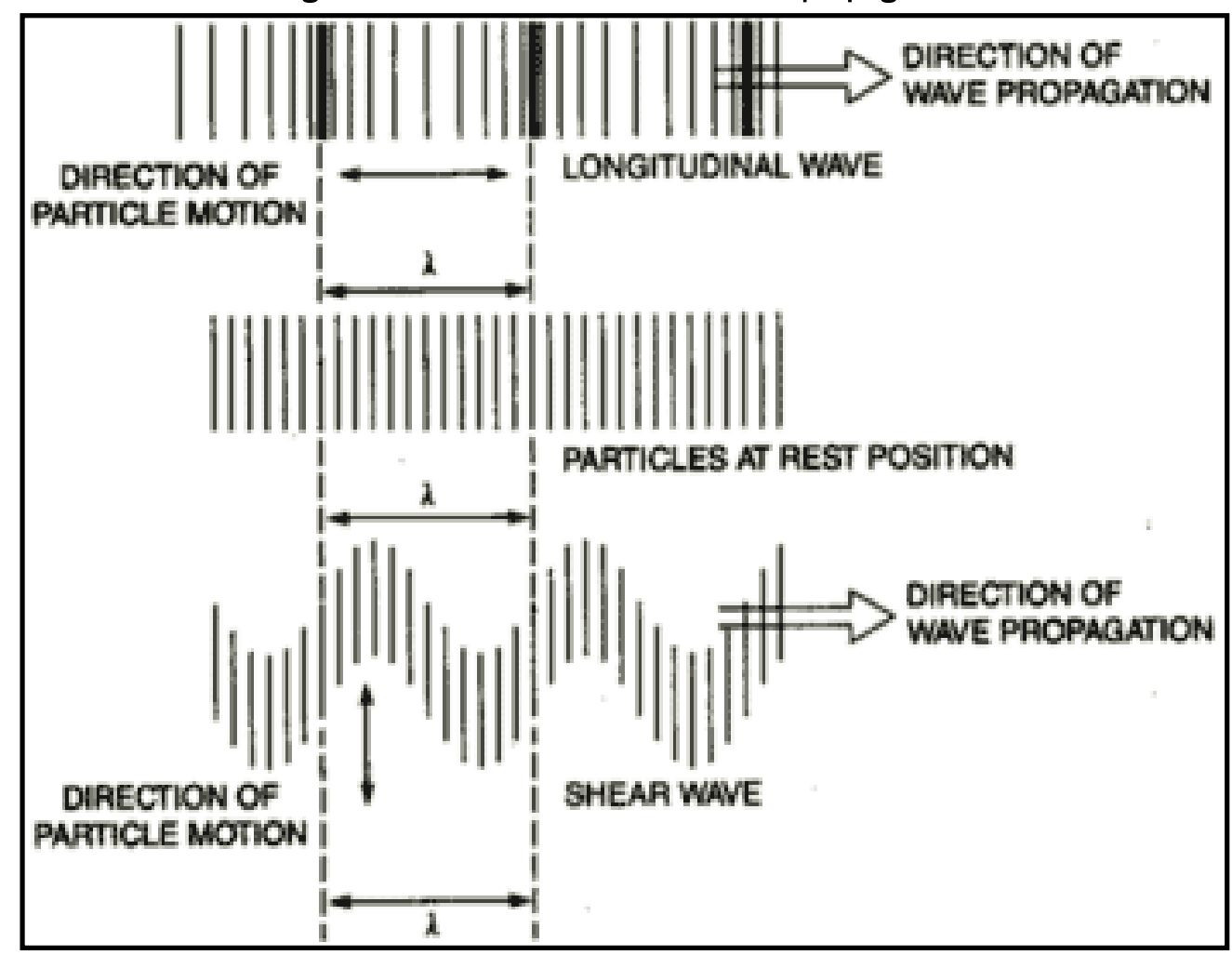


Carlyle et al. ${ }^{*}$ describe in detail the derivation and development of the acoustic measurement technique. The equations describing linear elastic deformation and ultrasonic wave velocity provide the basis for an equation describing how to determine Poisson's ratio inside a homogeneous material using pure ultrasonic measurements. The elastic deformation quantities include the P-wave or longitudinal modulus, shear modulus, and Poisson's ratio. The longitudinal modulus, $M$, relates the axial stress to the axial strain when the stress is applied in a uniaxial state:

$$
\sigma_{z z}=M \varepsilon_{z z}
$$

where $\sigma_{\mathrm{zz}}$ and $\varepsilon_{\mathrm{zz}}$ are the stress and strain, respectively, in the axial direction. The longitudinal velocity, $\mathrm{V}_{\mathrm{l}}$, is related to the longitudinal modulus and the shear velocity, $\mathrm{V}_{\mathrm{s}}$, is related to the shear modulus, $\mathrm{G}$, through the following equations:

$$
\begin{aligned}
& M=\rho V_{l}^{2} \\
& G=\rho V_{S}^{2}
\end{aligned}
$$

where $\rho$ is the density of the material. Possion's ratio, $v$, as a function of longitudinal and shear modulus can be written as:

$$
v=\frac{M-2 G}{2(M-G)}
$$

Equations 1-4 can be combined to give Poisson's ratio as a function of the longitudinal and shear velocities:

$$
v=\frac{V_{l}^{2}-2 V_{s}^{2}}{2\left(V_{l}^{2}-V_{s}^{2}\right)}
$$

\footnotetext{
* Carlyle, J. M., V. Hock, M. Mclnerney, and S. Morefield. 2004a. Mathematical Tension / Corrosion Prediction Models for Tainter Gate Tendon Rods. Final Report to the U.S. Army Corps of Engineers, Contract DACW42-03-P-0289. Champaign, IL: U.S. Army Engineer Research and Development Center, Construction Engineering Research Laboratory (ERDC-CERL); 2004b. Nondestructive Testing for Service Life Prediction of Reinforced Concrete Structures. Final Report to the U.S. Army Corps of Engineers, Champaign, IL: U.S. Army Engineer Research and Development Center, Construction Engineering Research Laboratory (ERDC-CERL).
} 
Equation 5 allows one to measure the dynamic Poisson's ratio as a function of acoustic velocities in a non-destructive manner. This equation is often used in geology to help determine stresses, but was not found to be applicable in determining the stress level in post-tensioned trunnion rods investigated in this work.

Although this equation was found to not successfully predict tension, another method may be employed where stress is related to second- and third-order elastic constants. The second-order constants are called Lamés parameters or Lamés constants and are a parameterization of the elastic moduli for homogeneous isotropic materials. Other elastic moduli can be expressed in terms of these constants. The third-order constants were introduced by Murnaghan (1937) and extended the linear elastic theory to include finite deformation in elastic isotropic materials. The longitudinal and shear acoustic velocities can be related to strain through the following equations involving Lamés constants and the third-order elastic constants (Bray and Stanley 1997):

$$
\begin{gathered}
\rho_{o} V_{l}^{2}=\lambda+2 \mu+(2 l+\lambda) \alpha+(4 m+4 \lambda+10 \mu) e_{1} \\
\rho_{o} V_{s}^{2}=\mu+(m+\lambda) \alpha+4 \mu e_{1}+2 \mu e_{2}-\frac{1}{2} n e_{3}
\end{gathered}
$$

where

$\rho_{0} \quad$ is the density in the unstressed state

$\mathrm{V}_{\mathrm{l}}$ is the longitudinal velocity

$\mathrm{V}_{\mathrm{s}}$ is the shear velocity

$\lambda$ and $\mu$ are the second-order Lamé's constants

$\mathrm{m}, \mathrm{l}$, and $\mathrm{n}$ are third-order elastic constants

$\mathrm{e}_{1}$ is the strain in the direction of the applied tension

$\alpha$ is the sum of the three principal strains given by:

$$
\alpha=e_{1}+e_{2}+e_{3}
$$

For a state of uniaxial stress where $\mathrm{e}_{1}=\varepsilon, \mathrm{e}_{2}=-v \varepsilon$, $\mathrm{e}_{3}=-v \varepsilon$, the longitudinal and shear velocities may be expressed as:

$$
\rho_{o} V_{l}^{2}=\lambda+2 \mu+[4(\lambda+2 \mu)+2(\mu+2 m)+2 v \mu(1+2 l / \lambda)] \varepsilon
$$




$$
\rho_{o} V_{s}^{2}=\mu+\left[4 \mu+m(1-2 v)+\frac{1}{2} n v\right] \varepsilon
$$

where Equation 9 used the following expression:

$$
v=\frac{\lambda}{2(\lambda+\mu)}
$$

The relative variation of the longitudinal and shear velocities with strain can be found using Equations 9 and 10 and are related to the acoustoelastic constants $\mathrm{L}_{\mathrm{l}}$ and $\mathrm{Ls}_{\mathrm{s}}$ according to:

$$
\begin{gathered}
\left.\frac{1}{V_{l 0}} \frac{d V_{l}}{d \varepsilon}\right|_{V_{l 0}}=2+\frac{(\mu+2 m)+v \mu\left(1+\frac{2 l}{\lambda}\right)}{\lambda+2 \mu}=L_{l} \\
\left.\frac{1}{V_{s 0}} \frac{d V_{s}}{d \varepsilon}\right|_{V_{s 0}}=2+\frac{m}{2(\lambda+\mu)}+\frac{v n}{4 \mu}=L_{s}
\end{gathered}
$$

where $d V_{1} / d \varepsilon$ and $d V_{s} / d \varepsilon$ were evaluated at the unstressed longitudinal and shear velocities given by $\mathrm{V}_{10}$ and $\mathrm{V}_{\mathrm{s} 0}$, respectively. This approximation is valid because the ultrasonic velocities do not change much over the entire elastic range. These equations can be solved for the differential strain, $\mathrm{d} \varepsilon$, which can be used in conjunction with Hooke's law, $\sigma=\mathrm{E} \varepsilon$, where $\mathrm{E}$ is Young's modulus, to find the change in stress, $\mathrm{d} \sigma$, related to the longitudinal and shear velocities:

$$
\begin{aligned}
& d \sigma=\frac{E}{L_{l}}\left(\frac{d V_{l}}{V_{l 0}}\right) \\
& d \sigma=\frac{E}{L_{s}}\left(\frac{d V_{s}}{V_{s 0}}\right)
\end{aligned}
$$

Thus, one only needs certain material constants to determine, either through measurement or modeling, the initial and change in longitudinal or shear wave velocity. 


\section{Ultrasonic Simulation and Measurement}

\subsection{Ultrasonic simulation}

A modeling and ray-tracing software package, Imagine3D, ${ }^{*}$ was used to model a 19-in. long plain steel rod measuring $1.25 \mathrm{in}$. diameter. This seemingly simple object actually involves a great deal of complexity due to beam divergence and mode conversion. ${ }^{\dagger}$ Figure 2 shows the experimental setup and the acoustic wave propagation simulation of the rod. The transducer is mounted on the left end of the rod. The acoustic energy travels down the rod, reflects off the right end of the rod, and travels back to the transducer. The ultrasonic beam from the transducer diverges (i.e., spreads) as it travels.

The lower plot in Figure 2 shows the beam divergence in which the blue rays emitting from the transducer have a clear conical shape. As the beam interacts with the longitudinal surface of the rod, it reflects and mode-con-

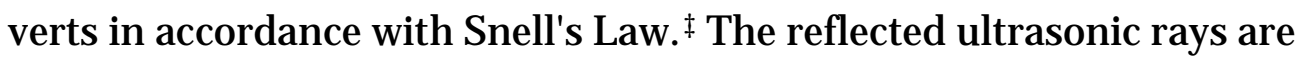
shown in green. Because of the number of rays involved, the reflected beam seems to fill the rod, but in reality each ray is traveling at an angle to the surface of the rod. Mode conversion is also taking place when the surface reflection occurs, and although the software simulation is producing rays that correspond to shear waves, they are not seen in the chosen simulation view.

Figure 3 shows the results of the beam divergence and mode conversion taking place inside the test rod when the simulator sums all of the received echoes. The rod is 19-in. long so, as expected, a strong echo appears at 19 in. from the transducer. This is produced by the longitudinal wave traveling straight down the rod, reflecting off the far end of the rod, and echoing back to the transducer.

\footnotetext{
* Note that the Microsoft Windows version of Imagine3D was discontinued by Impulse, Inc. after this modeling project was completed.

${ }^{\dagger}$ Conversion from a longitudinal to a shear wave and vice versa.

₹ According to Snell’s Law, the wave also refracts into the adjacent medium. If the adjacent medium is air, almost $100 \%$ (99.9964\%) of the wave will reflect back. If the adjacent medium is water, $87.83 \%$ is reflected. If the medium is concrete, only $36.60 \%$ will reflect. In our simple model, the adjacent medium is air so we are neglecting any loss of energy due to refraction of the wave at the longitudinal surface.
} 
Figure 2. Ultrasonic instrument showing received signal from transducer mounted on left end of rod (top); simulation of signal propagation within the rod (bottom).

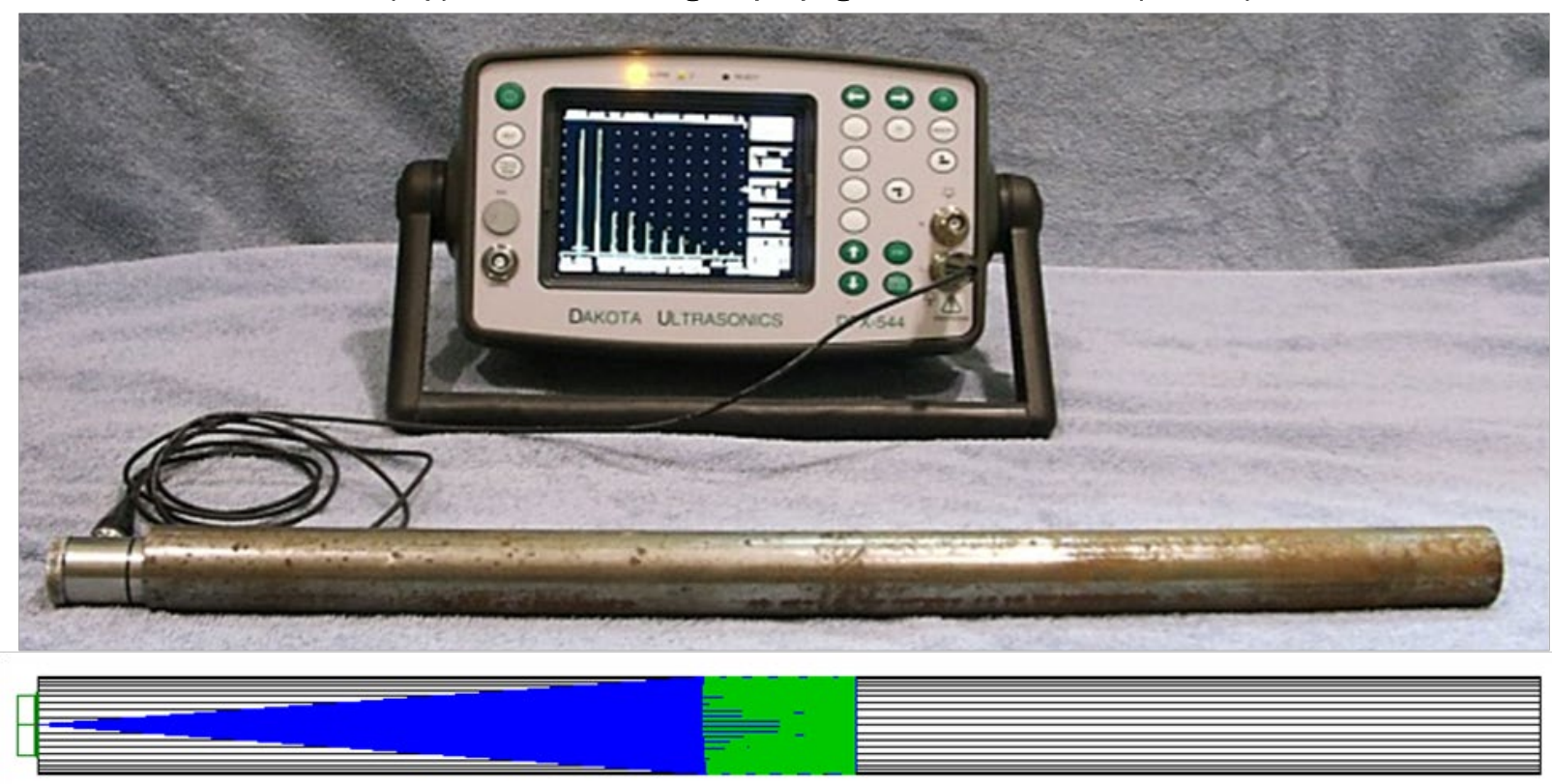

Figure 3. Simulation of echoes in sample rod (top) and actual screen shot from ultrasonic instrument display (bottom).

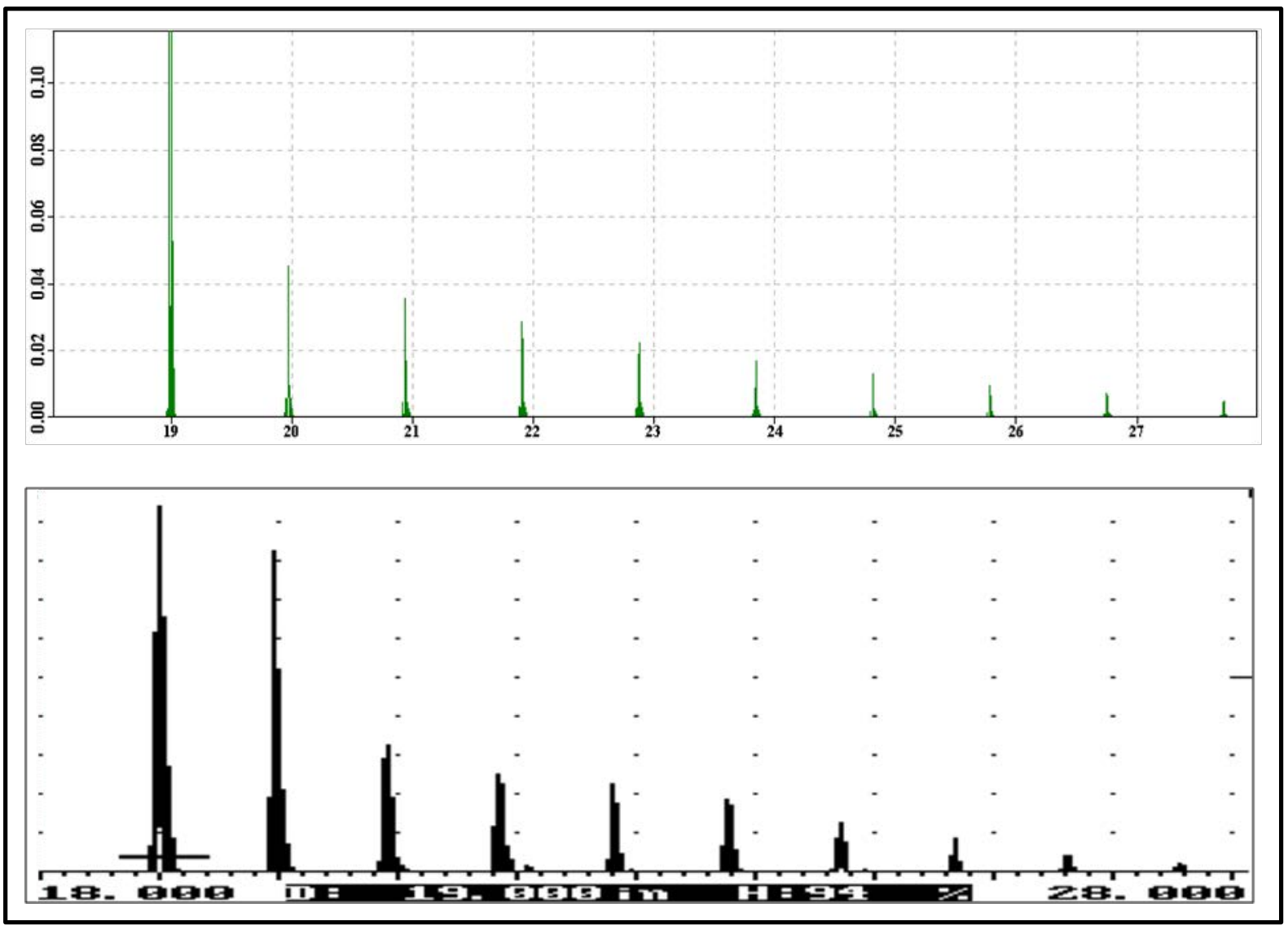


Note that other echoes appear after 19 in. These are caused by mode conversion of the original longitudinal ultrasonic beam into shear waves as the diverging beam interacts with the longitudinal surface of the rod. Since shear waves travel in steel about half as fast as longitudinal waves, when they arrive back at the transducer, they will lag behind the faster longitudinal waves. The agreement between the simulation and the result obtained from the measurement is very good.

In addition to placing all the echoes at the proper distance, the modeling software also did a good job of calculating the expected amplitude for the echoes. The only significant disagreement between the result obtained from the measurement and simulation appears in the echo at $20 \mathrm{in}$. The measurement recorded a stronger echo than predicted by the simulation. All the other echoes, from about 21 to $28 \mathrm{in}$., agree well between measurement and simulation.

\subsection{Tension measurements using ultrasound}

The key to computing tension in a component is to obtain the necessary ultrasonic measurements with a high degree of accuracy. There are two ways to do this: (1) modeling software can be used to compute unknown values from measured values; (2) all values can be measured directly.

The tension measurement model consists of theoretical equations together with an accurate ultrasonic echo model. A material's bulk stress, $\sigma$, is calculated in terms of ultrasonic quantities. These equations permit us to measure the tension in an embedded steel rod, such as a tainter gate anchor rod, by measuring ultrasonic properties. The physical changes produced by tension in a rod can be determined using the isotropic version of Hooke's Law:

$$
E=\sigma_{\mathrm{zz}} / \varepsilon_{\mathrm{zz}}
$$

Stress, $\sigma$, is force (tension) divided by area, while strain, $\varepsilon$, is change in length divided by the original length. The following equation shows the re lationship for the length change that results from a given tension in a rod: 


$$
\Delta L=F L / A E
$$

where:

$$
\begin{aligned}
& F=\text { applied force } \\
& L=\text { original length of the rod } \\
& A=\text { circular area of the rod } \\
& E=\text { Young's modulus. }
\end{aligned}
$$

The change in length for various applied loads to a 19-in. long x 1.25 in. diameter steel rod is calculated using Equation 17 and listed in Table 1.

Table 1. Calculated load-induced length change in a $19 \times 1.25$ in. (diameter) steel rod.

\begin{tabular}{|l|c|}
\hline \multicolumn{1}{|c|}{ Load (lb) } & Length Change (in.) \\
\hline 100 pounds & 0.000525 in. \\
\hline 500 pounds & 0.002627 in. \\
\hline 1000 pounds & 0.005253 in. \\
\hline 5000 pounds & 0.026267 in. \\
\hline 10000 pounds & 0.052534 in. \\
\hline
\end{tabular}

From the data in Table 1, the modeling software can be used to predict the exact position of the ultrasonic echoes that will result when the length of the example rod is increased by these lengths. Thus, the effect of tension on ultrasound echoes in the steel bar can be observed. This accomplishes two things; it permits us to see: (1) the magnitude change of any given tension state and (2) the effect of tension on any particular echo. The first point is important because it allows us to determine the precision with which the ultrasonic velocity measurements will need to be conducted.

The echoes shown in Figure 4 are produced in the modeling software by specifying the wave modes that can exist in the rod. The first echo, at 19 in., is the result of a longitudinal wave propagating down the length of the rod and another longitudinal wave reflecting off the far end and returning to the transducer. This can be seen clearly in the top and bottom plots of Figure 4, in which the blue rays are the longitudinal wave propagating down the length of the rod and the green rays correspond to this longitudinal wave, reflecting off the far end and returning to the transducer. There are no shear waves in the first echo because the shear waves are created by 
mode conversion of the reflecting longitudinal wave, and because they travel at about half the velocity of the longitudinal waves.

Figure 4. Model of $19 \times 1.25$ in. (diameter) steel rod showing how first echo (green rays) and second echo (magenta) form (top); model showing how the first echo (green rays) and third echo (gray rays) form (bottom).

The second, third, and higher echoes consist of a mix of longitudinal waves and shear waves. The top plot of Figure 4 also shows how the second echo is created. A longitudinal wave (blue) propagates down the rod, a reflected longitudinal wave (green) reflects off the far end, and a reflected shear wave (dark purple) is created when the reflected longitudinal wave modeconverts at the longitudinal surface of the rod. Another reflected longitudinal wave (magenta) is created when this shear wave mode-converts on the opposite surface of the rod. This last longitudinal wave returns to the transducer to create echo 2 . This event can be expressed with the following equation for the time of echo $2\left(t_{e} 2\right)$ :

$$
t_{e 2}=\frac{x_{2}}{v_{1}}+\frac{x_{3}}{v_{1}}+\frac{x_{4}}{v_{\mathrm{s}}}+\frac{x_{5}}{v_{1}}
$$

where $v_{l}$ and $v_{s}$ are the longitudinal and the shear wave velocities, respectively, and $\mathrm{x}_{2}, \mathrm{x}_{3}, \mathrm{x}_{4}$, and $\mathrm{x}_{5}$ are the path lengths for their respective rays in the rod.

The bottom plot of Figure 4 shows how the third echo is created. A longitudinal wave (blue) propagates down the rod and a reflected longitudinal wave (green) reflects off the far end. A reflected shear wave (dark purple) is created when the reflected longitudinal wave mode-converts at the longitudinal surface of the rod, and another reflected shear wave (magenta) is created when the first reflected shear wave reflects off the opposite side of the rod. Finally, a reflected longitudinal wave (gray) is created when this second shear wave mode-converts on the opposite side of the rod. This last longitudinal wave returns to the transducer to create echo 3 . This can be expressed with the following equation for the time of echo 3, te3: 


$$
t_{e 3}=\frac{x_{2}}{v_{1}}+\frac{x_{3}}{v_{1}}+\frac{x_{4}}{v_{s}}+\frac{x_{6}}{v_{s}}+\frac{x_{7}}{v_{1}}
$$

where $\mathrm{v}_{\mathrm{l}}$ and $\mathrm{v}_{\mathrm{s}}$ are the longitudinal and the shear wave velocity, respectively, and $\mathrm{x}_{2}, \mathrm{x}_{3}, \mathrm{x}_{4}, \mathrm{x}_{6}$, and $\mathrm{x}_{7}$ are the path lengths for their respective rays in the rod. Note that $\mathrm{x}_{2}, \mathrm{x}_{3}$, and $\mathrm{x}_{4}$ are common to echoes 2 and 3.

An equation for the velocity of the shear wave as a function of the velocity of the longitudinal wave and the echoes in the rod is obtained by subtracting Equation 18 from Equation 19:

$$
v_{\mathrm{s}}=\left(v_{1} \times x_{6}\right) /\left(v_{1} \times\left(t_{\mathrm{e} 3}-t_{\mathrm{e} 2}\right)+x_{5}-x_{7}\right)
$$

The modeling software can compute the values of the four path lengths $\mathrm{x}_{4}$, $\mathrm{x}_{5}, \mathrm{x}_{6}$, and $\mathrm{x}_{7}$. The software can also provide the times of the two required echoes, te2 and te3. These computed quantities, in combination with the measured value of the longitudinal wave velocity, $\mathrm{vl}$, are all that is needed to obtain the last desired quantity, vs. To obtain the change in tension in the rod, one uses Equations 14 and 15 and the measured longitudinal velocity, $\mathrm{vl}$, and the computed shear velocity, vs.

The modeling software can be used to determine values of other parameters that are unknown or difficult to measure.

\subsection{Verification testing}

Laboratory verification was conducted using plain steel rods that were about 90 -in. long and had 1.25-in diameters. The rods were placed under tension in a load frame. The setup permitted free access to the rod ends for ultrasonic transducer placement. The velocities of the longitudinal and shear waves were measured at various tensions using individual transducers in conjunction with an ultrasonic flaw detector. In these experiments, good agreement was obtained between direct tension measurement and the tension calculated from acoustic measurements. Figure 5 shows the experimental setup; Figure 6 shows the transducers, flaw detector, and voltmeter for measuring the strain. 
Figure 5. Pictures of the experimental setup for measuring tension and longitudinal and shear velocities.

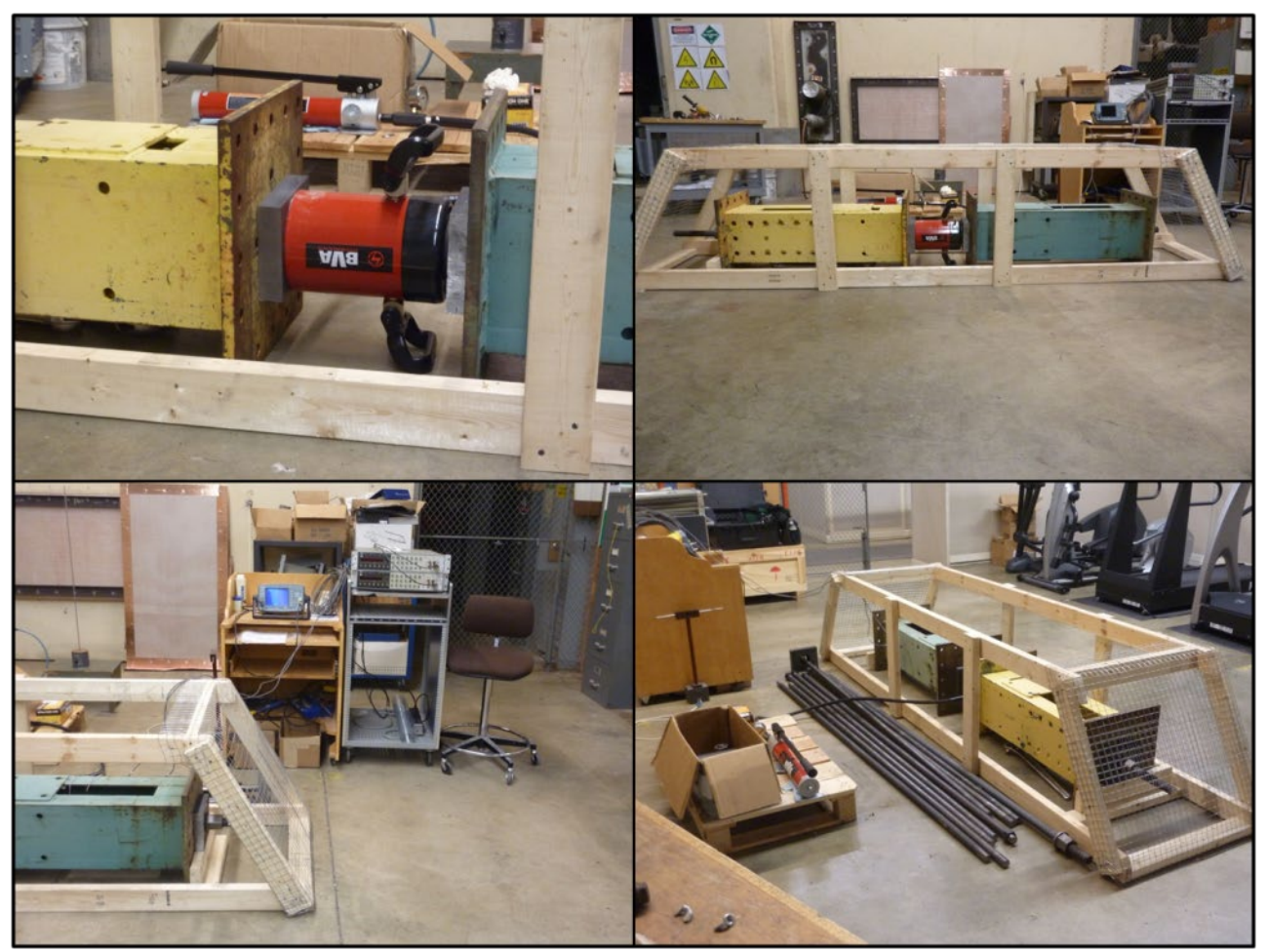

Figure 6. Pictures of the (upper left) transducers, (lower left) digital voltmeter used to readout the strain from the strain gauge, and (right) the flaw detector used in the experiments.

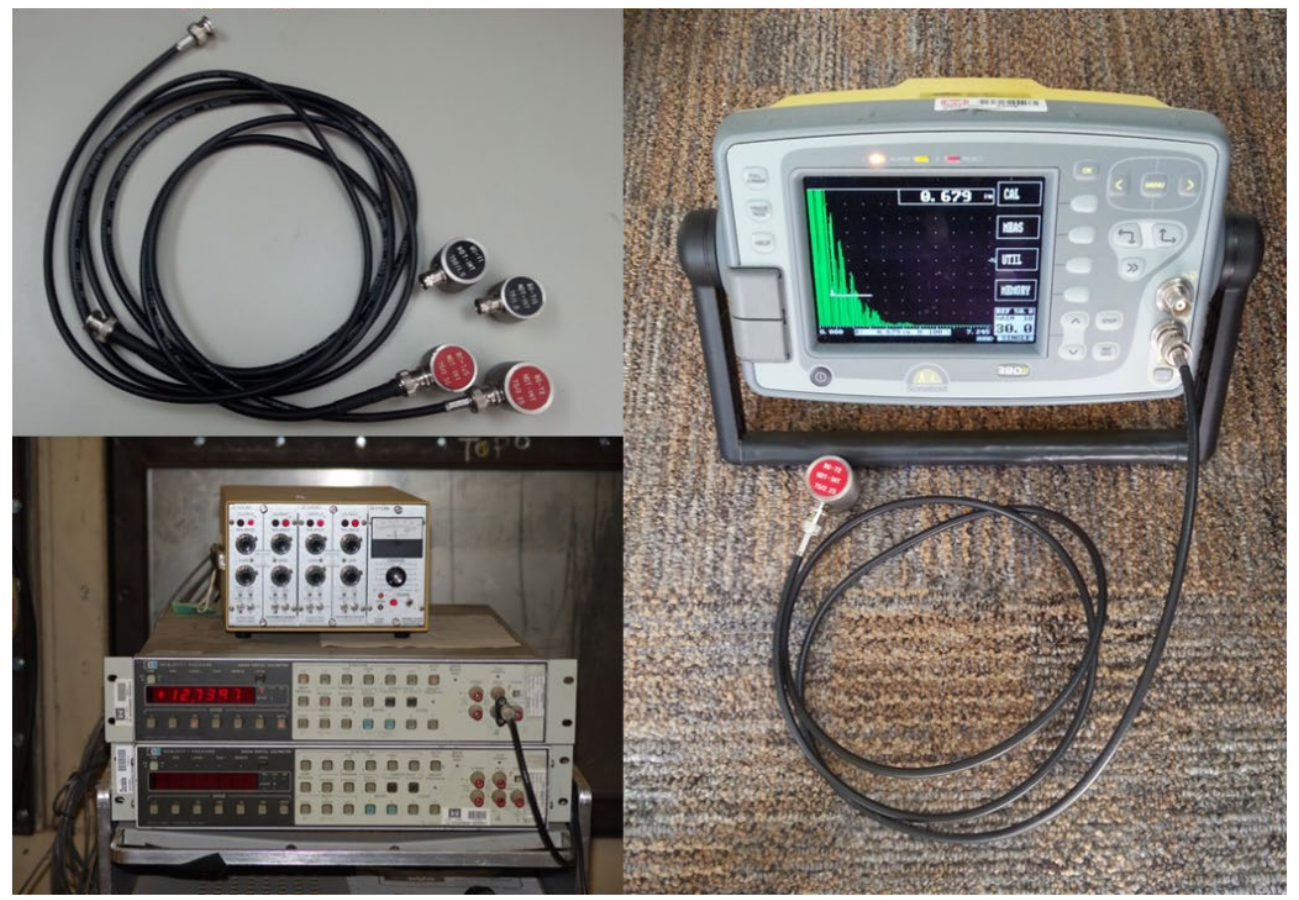


Rods of five different steel types were tested including: 12L14, 1018, 1144, A36, and DYWIDAG THREADBAR ${ }^{*}$ at a transducer frequency of $1 \mathrm{MHz}$ and 2.25 MHz. At least three runs were performed for each rod and frequency. Each rod and was placed in the load frame and a nut was threaded on both ends of the rod. A manual pump (BVA Hand Pump [10000 PSI], BVA Single Acting, Hollow Hole Cylinder [HC6003T]) was used to apply load to both ends of the rod resulting in tension. For each rod and at both transducer frequencies, longitudinal velocity, shear velocity, load, displacement, and strain were measured at about 10 stress levels from zero stress up to near the yield stress. The experiment was surrounded by a protective cage to prevent injury if the rod failed.

The stress was calculated according to Hook's law using the measured strain from the applied strain gauges. The strain was measured using a strain gauge (Micro Measurements Group, Precision Strain Gauge, CEA-06250UW-350), which was placed about $2.5 \mathrm{ft}$ from one end of the rod, and read out with a digital voltmeter (2120B Strain Gauge Conditioner, Hewlett Packard 3455 A Digital Voltmeter). The longitudinal and shear velocities were measured using either a $1 \mathrm{MHz}$ or $2.25 \mathrm{MHz}$ transducer (NDT International [BC-72, BC-72 S, BC-71, and BC-71 S]), which was held to the rod with the proper ultrasonic couplant. The time of flight was measured using an ultrasonic flaw detector (Sonatest $380 \mathrm{M}$ ) and then the length of the rod was divided by one half of the time of flight to obtain the appropriate wave velocity. Figure 7 shows the results of the longitudinal and shear velocity plotted as a function of stress. The longitudinal velocity was observed to decrease with increasing stress while the shear velocity was observed to remain about the same or to slightly decrease with increasing stress.

To compare these experimental results to the theory given by Equations 12 and 13, the longitudinal and shear velocities were normalized and plotted as a function of strain shown in Figure 8 and in Appendix B. These curves all followed a linear trend as the theory predicted. A linear curve was fit to these plots to extract the parameters $\mathrm{Ll}$ and Ls, which are given by the average slopes of the fits for each type of steel rod measured.

* DYWIDAG THREADBAR ${ }^{\circledR}$ is a steel bar marketed by DSI (DYWIDAG Systems International). THREADBAR $\circledast$ are hot rolled and proof stressed alloy steel conforming to ASTM A722 CAN/CSA (G279-M1982). The bars have a continuous rolled-in pattern of thread-like deformations along their entire length. The strength of the DYWIDAG anchorages and couplers exceeds the requirements of ACI 318 and the PTI Acceptance Standards for Post-Tensioning Systems. 
Figure 7. Plots of the measured longitudinal and shear velocities as a function of applied stress for three independent trial runs of steel 1018 using a $2.25 \mathrm{MHz}$ transducer. The dashed line in (a) is the theoretically derived fitting function.

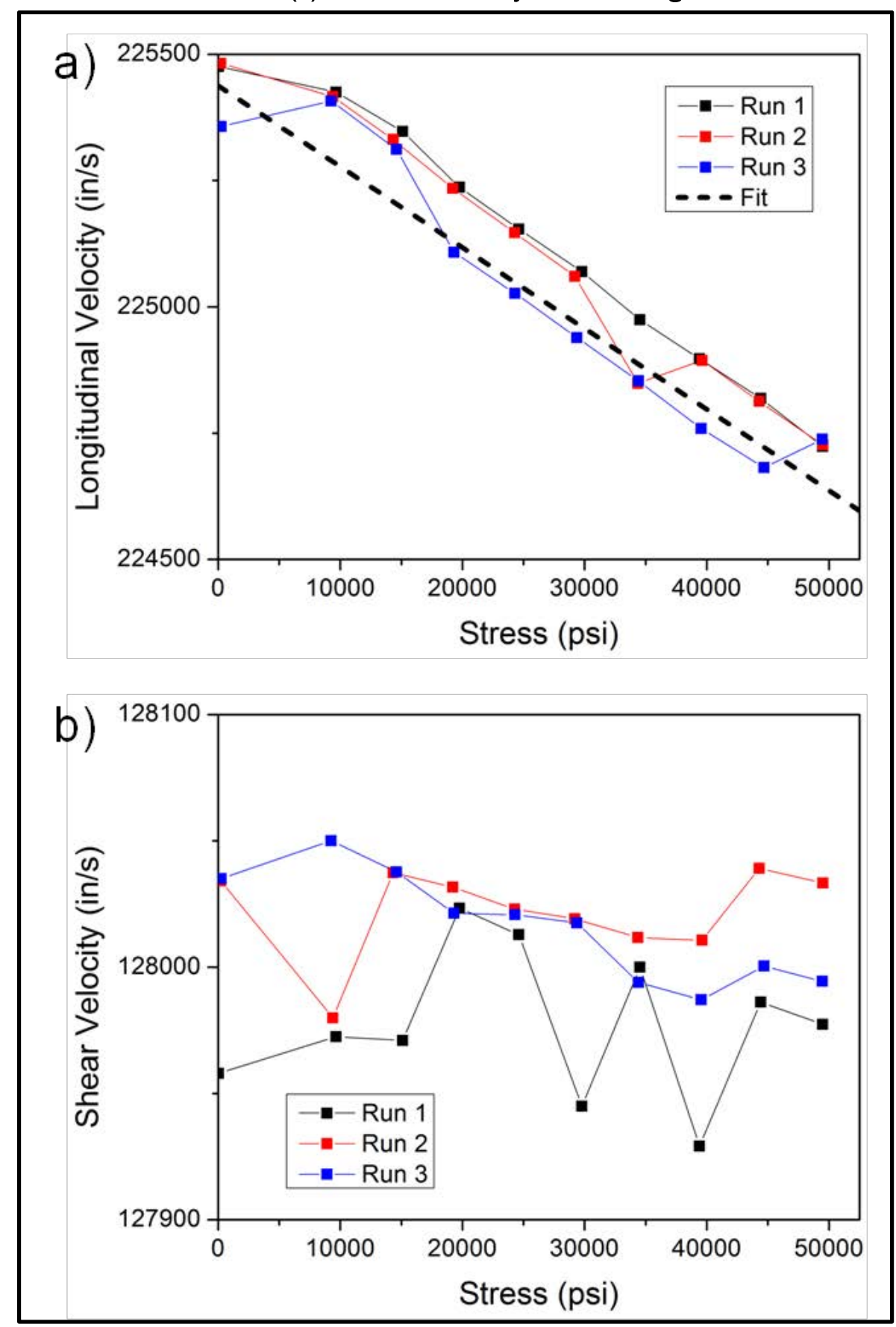

The range of the Lu parameter for the steel rods tested was -1.54 to -2.73 , which falls partly in the range of literature values for commonly used steels of -2.10 to -2.45 (Bray 2000). The range of the Ls parameter of the rods tested was -0.71 to 0.2 . The value of the $\mathrm{L}_{\mathrm{s}}$ parameter was comparable to the noise/ error level of 
the experimental setup and was not considered suitable for data fitting. Only the Li value was used to compare to the experimental data.

For 1018 steel with a $2.25 \mathrm{MHz}$ transducer, the values for the parameters $\mathrm{L}$ and $L_{s}$ were -2.11 and -0.08 , respectively. The $L_{l}$ parameter can be used in conjunction with Equation $14\left(d \sigma=\frac{E}{L_{l}}\left(\frac{d V_{l}}{V_{l 0}}\right)\right)$ to predict the stress value for a given change in longitudinal velocity. There are two other quantities that must be known for a prediction of the stress value in the rod. Both Young's modulus and the original or unstressed longitudinal velocity must be known. Most steels have a Young's modulus of about 3x107 psi. Young's modulus for 1018 steel is $2.97 \times 10^{7} \mathrm{psi}^{*}$ and the average value of the measured longitudinal velocities in the unstressed state for this experiment was $225,438 \mathrm{in} / \mathrm{s}$. Thus the equation that predicts stress as a function of change in longitudinal velocity for 1018 steel is: $\sigma=-62.4\left(\mathrm{dV}_{\mathrm{l}}\right)$, where $\sigma$ is in psi and $\mathrm{dV}_{\mathrm{l}}$ is in in/s and referenced to the unstressed state. This fitting function is plotted in Figure 8(a) and is an excellent fit to the three experimental measurements. For this model to be an accurate prediction of the actual stress level in the rods, the longitudinal velocities must be measured with great precision. The variation in the data was lower for the $2.25 \mathrm{MHz}$ transducers than for the $1 \mathrm{MHz}$ transducers as can be seen in Figures B-1 and B-2 of Appendix B. It is thus recommended that measurements of the ultrasonic parameters takes place at $2.25 \mathrm{MHz}$ and higher frequencies be explored.

\subsection{Corrosion Prediction using Ultrasound}

In addition to tension, the Imagine3D software was used to simulate the addition of a small corrosion pit to the steel rod. We simulated a corrosion pit by removing an ellipsoid of material from the top of the steel rod. (Note that this is the same as assuming that the ellipsoid is filled with air.) The ellipsoid extended $0.1 \mathrm{in}$. into the rod, had a diameter across the ultrasonic beam of $0.1 \mathrm{in}$., and had a diameter along the ultrasonic beam of $0.25 \mathrm{in}$. Appendix A presents this analysis. This modeling effort was conducted strictly as a proof of concept; no verification via laboratory measurements or field testing was performed.

\footnotetext{
* AZO Materials - AISI 1018 Mild/Low Carbon Steel, http://www.azom.com/article.aspx?ArticleID=6115.
} 
Figure 8. Plots of the scaled longitudinal and shear velocities as a function of strain for independent trial runs of steel 1018 using a $2.25 \mathrm{MHz}$ transducer. Each set of data is fit to a linear curve and the average slope, given by $<\mathrm{m}>$ is recorded.

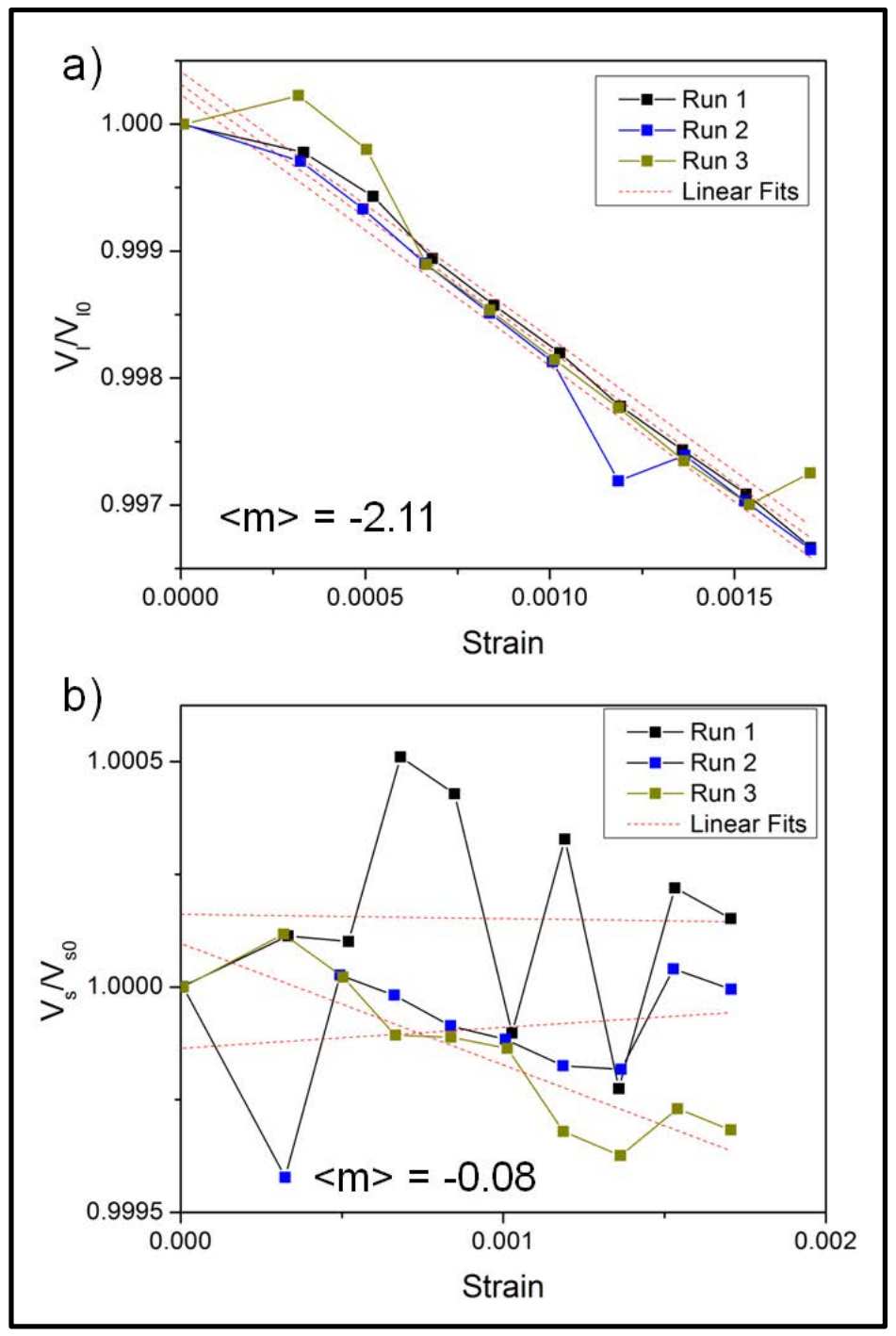




\section{Field Testing Acoustic Nondestructive Technique}

Field tests of the acoustic NDT technique have been completed at three U.S. Army Corps of Engineers dams, one each in Georgia, Oklahoma, and Illinois. Measurements of the ultrasonic velocities were taken on trunnion anchorage anchor rods. Trunnion anchorages are large concrete blocks that anchor the tainter gate trunnions to the dam. (The trunnions are the giant pivots to which the tainter gates are attached.) The concrete anchorages are bolted to the dam with large steel rods that extend through the anchorages into the piers of the dam.

Figure 9 shows a photograph of tainter gates and trunnion anchorages at one of the test locations. In this picture, the anchor rods are located behind the pairs of vertical rectangular steel enclosures about halfway up from the water line.

Figure 10 shows the design of these tainter anchorages, which is described in Engineer Manual (EM) 1110-2-2702 (HQUSACE 2000). This design was mandated by Headquarters, U.S. Army Corps of Engineers in the 1960s and has been adopted by other government agencies and by industry. It offered advantages over conventional steel beam-and-girder designs, but a significant problem is that the anchor tendons (i.e., rods) are inaccessible for expedient inspection and repair.

A complete post-tensioned anchorage system includes tendons (bars/ rods or strands), anchorage devices or bearing plates, ducts, end caps, grout tubes, couplers, anchorage zones, and a corrosion protection system. Figure 11 shows these components.

Ducts encase the tendons to separate them from the surrounding pier and abutment concrete and allow tensioning after the concrete has cured. The ducts also protect anchors during placement of concrete and act as part of the corrosion-prevention system. The tendons are post-tensioned, following installation of the concrete anchorages. 
Figure 9. Typical tainter gates and trunnions.

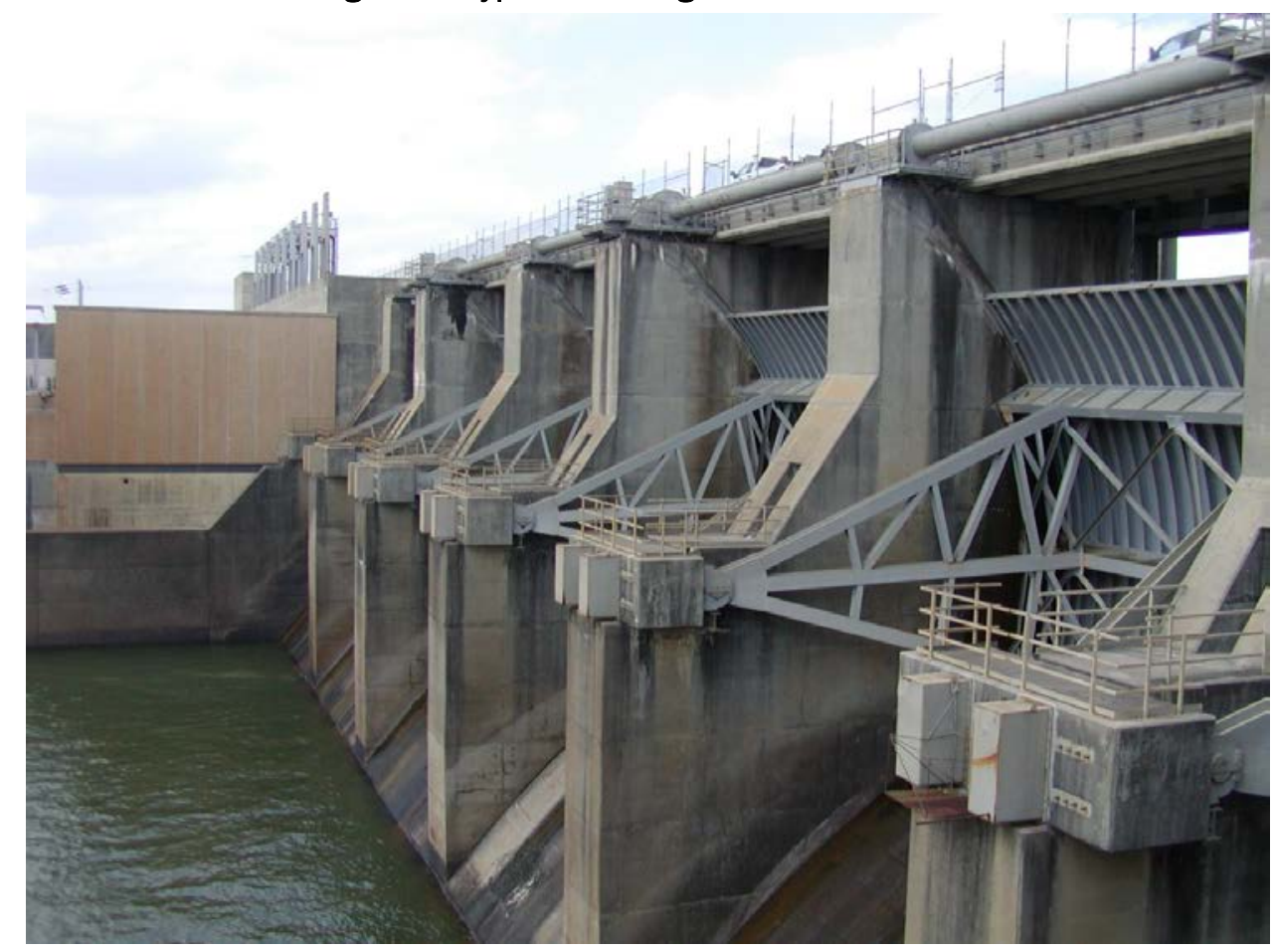

Figure 10. Trunnion anchorage design.

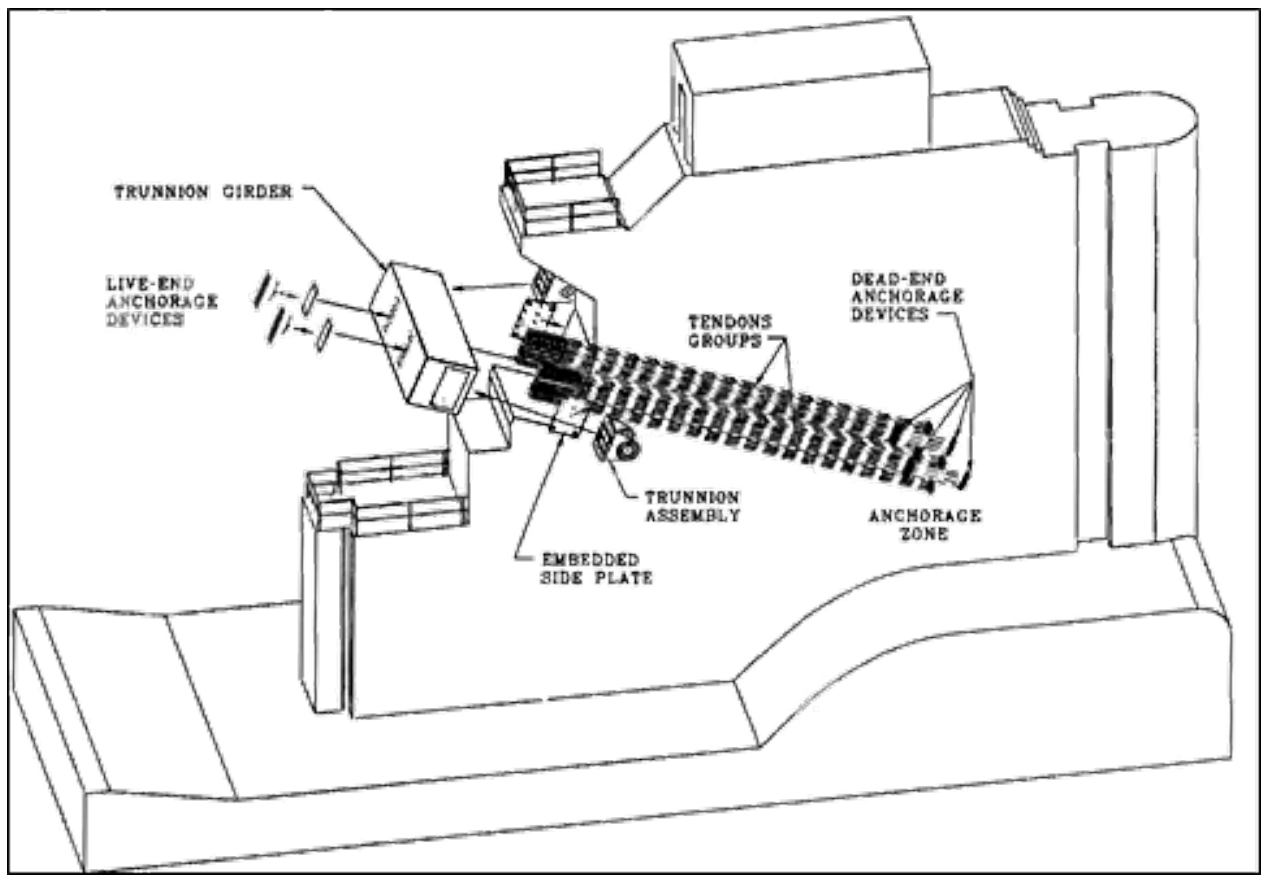

Source: EM 1110-2-2702 (HQUSACE 2000). 
Figure 11. Typical post-tensioned anchorage system.

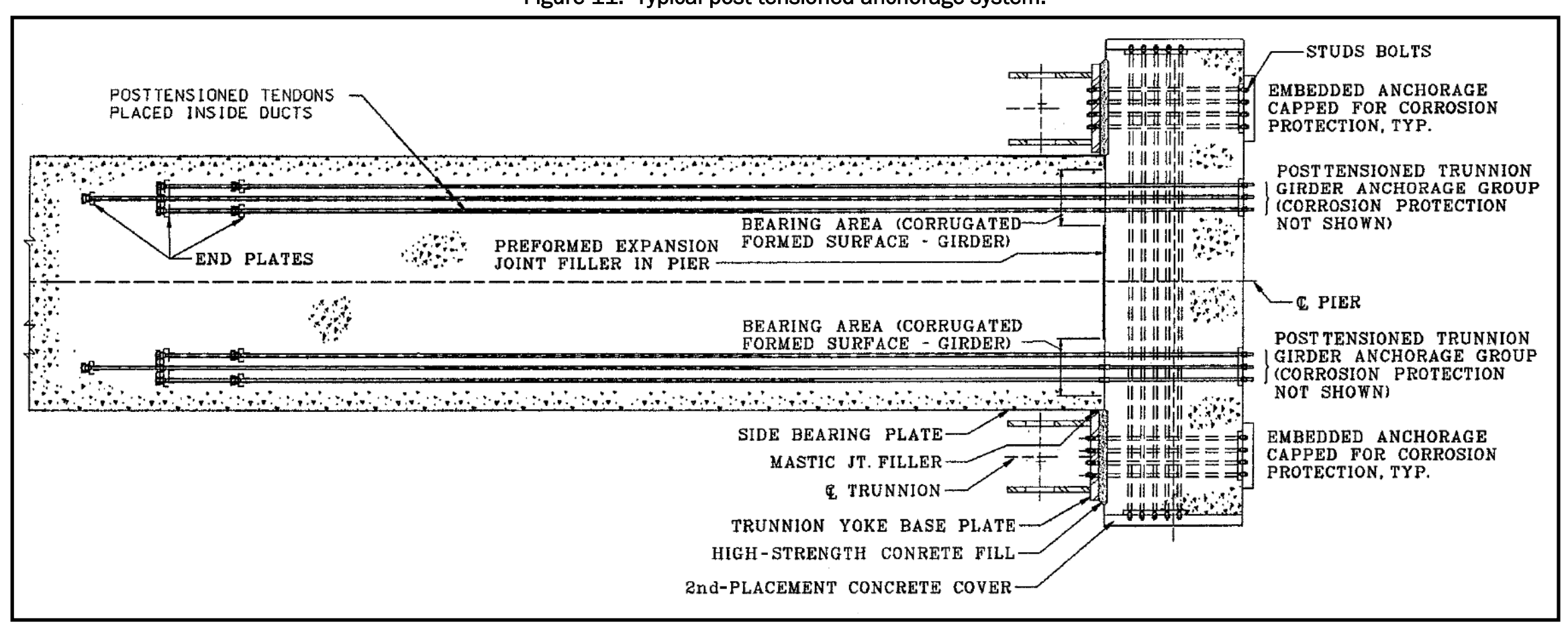

Source: EM 1110-2-2702 (HQUSACE 2000). 
The corrosion-prevention system for tendons consists of tendon ducts, duct fittings, connections between ducts and anchorages, grout tubes, end caps, and filler. The filler material can be either grease or grout. The filler material encapsulates the tendon to prevent corrosion, and is injected after tensioning.

Over the past few years, several tendon (rod) failures have been noted. Figure 12 (left) shows several failed rods, one that has penetrated a cover and two that have dimpled the covers from the inside upon fracturing. These covers are one barrier to inspecting the rods. They are large and made of heavy gauge steel, and are secured with many bolts or sometimes even peripherally welded to the anchorage. There are no designed inspection hatches, although some have been retrofitted during recent maintenance. As can be seen in Figure 9, these covers are located where access is difficult.

Figure 12. Failed anchor rod penetrating cover, showing one dimple in each cover indicating failed rods that did not penetrate (left); cover removed exposing anchor rods prior to testing (right).
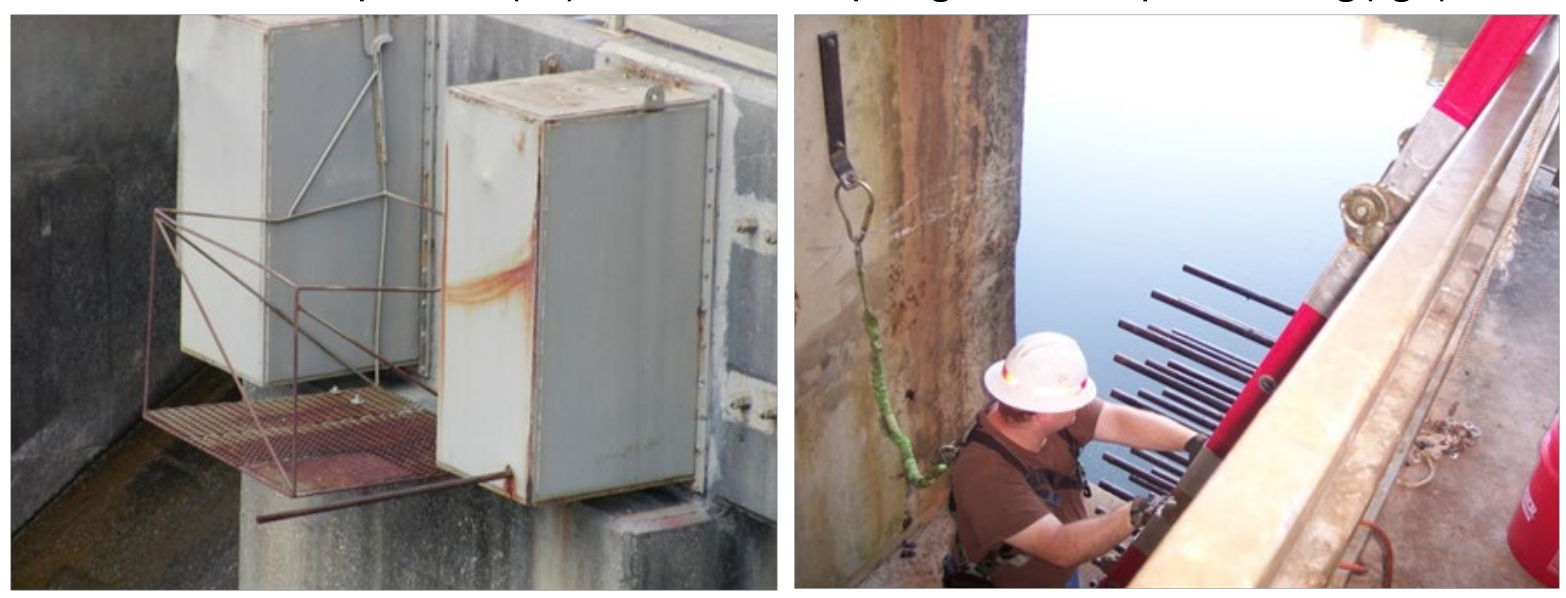

Figure 12 (right) shows the ends of the rods where the cover has been removed for anchor rod maintenance and testing.

The first field test was conducted at Keystone Dam in Oklahoma. This dam has an atypical design as both ends of the rods are exposed. A gallery runs through the dam, providing access to the lower ends of the rods. The rods are also secured with threaded nuts so they can be easily retensioned. Grease is used as filler in the tendon ducts.

Twenty rods (out of 56 total) on a single pier were randomly selected for testing. All rods were $50 \mathrm{ft}$ long. All rod ends were smoothed on the inside of 
the dam. On the outside, only 10 ends were smoothed, leaving 10 with rough, flame-cut ends. Consequently, 10 rods had good ultrasonic reflectors on the far end (testing was done from the inside) and 10 had poor far-end ultrasonic reflectors. Testing showed that longitudinal ultrasound propagated through only six of the 20 rods (five were smooth on both ends, while one was smooth on only one end). Shear ultrasound propagated through only one rod (which was smooth on both ends). It is not known why the two modes of ultrasound propagated through only one of the rods.

Previous laboratory experiments on a failed 36-ft rod removed from another dam showed there should have been enough signal strength (with a threshold in excess of $46 \mathrm{~dB}$ ) to interrogate a $50 \mathrm{ft}$ rod. The system was calibrated using that rod. Some signal improvements were obtained onsite by careful polishing of the rod ends. Possible explanations for the increased attenuation are that the composition of these rods is slightly different from the test rod, and that the signal was dispersing into the filler material in contact with the rods. The laboratory rod tests were performed in air.

The second field test was conducted at West Point Dam in Georgia (Figures 9 and 12). On one pier, 38 rods measuring 38 to $48 \mathrm{ft}$ were tested. The filler material was grease. Both longitudinal and shear ultrasonic signals were obtained for all rods. The measured longitudinal and shear velocities are recorded for Block E West and Block E East in Tables 2 and 3, respectively.

Table 2. Longitudinal and shear velocity in Block E West.

\begin{tabular}{|l|c|c|c|c|c|c|}
\hline \multirow{2}{*}{ Row } & \multicolumn{2}{|c|}{ Rod Col. A } & \multicolumn{2}{c|}{ Rod Col. B } & \multicolumn{2}{c|}{ Rod Col. C } \\
\cline { 2 - 7 } & $V_{I}$ (in/s) & $V_{s}$ (in/s) & $V_{I}$ (in/s) & $V_{s}$ (in/s) & $V_{I}$ (in/s) & $V_{s}$ (in/s) \\
\hline 1 & 237,262 & 128,822 & 236,448 & 128,959 & 235,746 & 129,236 \\
\hline 2 & 236,840 & 128,781 & 236,891 & 129,292 & 236,281 & 128,892 \\
\hline 3 & 235,942 & 128,737 & 236,097 & 128,723 & 235,515 & 128,675 \\
\hline 4 & 235,773 & 129,233 & 236,583 & 128,944 & 235,071 & 128,758 \\
\hline 5 & 237,294 & 128,862 & 235,562 & 128,758 & 235,784 & 129,286 \\
\hline 6 & 236,721 & 128,880 & 236,849 & 128,870 & 236,226 & 129,236 \\
\hline 7 & 236,794 & 128,925 & 236,188 & 128,746 & 236,955 & 128,877 \\
\hline 8 & 236,205 & 128,827 & 236,220 & 128,862 & 236,400 & 128,898 \\
\hline 9 & 237,653 & 128,984 & 236,978 & 128,953 & 236,785 & 128,847 \\
\hline 10 & 238,531 & 129,024 & 237,627 & 128,977 & 238,161 & 128,910 \\
\hline
\end{tabular}


Table 3. Longitudinal and shear velocity in Block E East.

\begin{tabular}{|l|c|c|l|l|}
\hline \multirow{2}{*}{ Row } & \multicolumn{2}{|c|}{ Rod Col. A } & \multicolumn{2}{c|}{ Rod Col. B } \\
\cline { 2 - 5 } & $V_{\boldsymbol{V}}(\mathrm{in} / \mathrm{s})$ & \multicolumn{1}{|c|}{$\boldsymbol{V}_{\boldsymbol{s}}(\mathrm{in} / \mathbf{s})$} & $V_{\boldsymbol{V}}(\mathrm{in} / \mathbf{s})$ & $V_{\boldsymbol{s}}(\mathrm{in} / \mathbf{s})$ \\
\hline 1 & 235,123 & 128,251 & 236,343 & 128,337 \\
\hline 2 & 234,257 & 128,020 & 234,837 & 128,182 \\
\hline 3 & 236,525 & 128,328 & 236,741 & 128,189 \\
\hline 4 & 235,855 & 128,396 & Broken & Broken \\
\hline
\end{tabular}

There was a variance in the velocity measurements. The average measured value was $236,407 \mathrm{in} / \mathrm{s}$ for longitudinal velocity and $128,797 \mathrm{in} / \mathrm{s}$ for shear velocity. The standard deviation of the measured values is $882 \mathrm{in} / \mathrm{s}$ and $316 \mathrm{in} / \mathrm{s}$, respectively. One would expect the rods to change tension uniformly and without such extreme variance in the ultrasonic velocities. We have high confidence in our measurements and attribute this larger than expected variation to the uncertainty in the length of the rods since the rod lengths were taken from engineering drawings that reported to lengths in $1 / 4$-in. segments suggesting an uncertainty of about $1 / 8$ in. Unlike the Oklahoma test, the acoustic signals were easily detectable. The difficulties in the Oklahoma test were because of shear wave attenuation. (The attenuation of the shear wave is four to 10 times greater than that of the longitudinal wave.) We were able to obtain longitudinal and shear wave measurements on all 37 rods but obtaining a shear measurement was difficult for a few of the $48 \mathrm{ft}$ rods. This led us to conclude that our current instrumentation and transducers are suitable for use on rods up to $45 \mathrm{ft}$ in length.

The third field test was conducted at Kaskaskia Lock and Dam in Illinois (Figure 13). Figure 14 shows the rod ends. Of the 112 rods in the sample, 42 measured $42 \mathrm{ft}$ long, 42 were $46 \mathrm{ft}$, and 28 were $50 \mathrm{ft}$. All rods had diameters of $1.25 \mathrm{in}$. and the filler material was grout. 
Figure 13. Site of third field test, Kaskaskia Lock and Dam, Illinois.

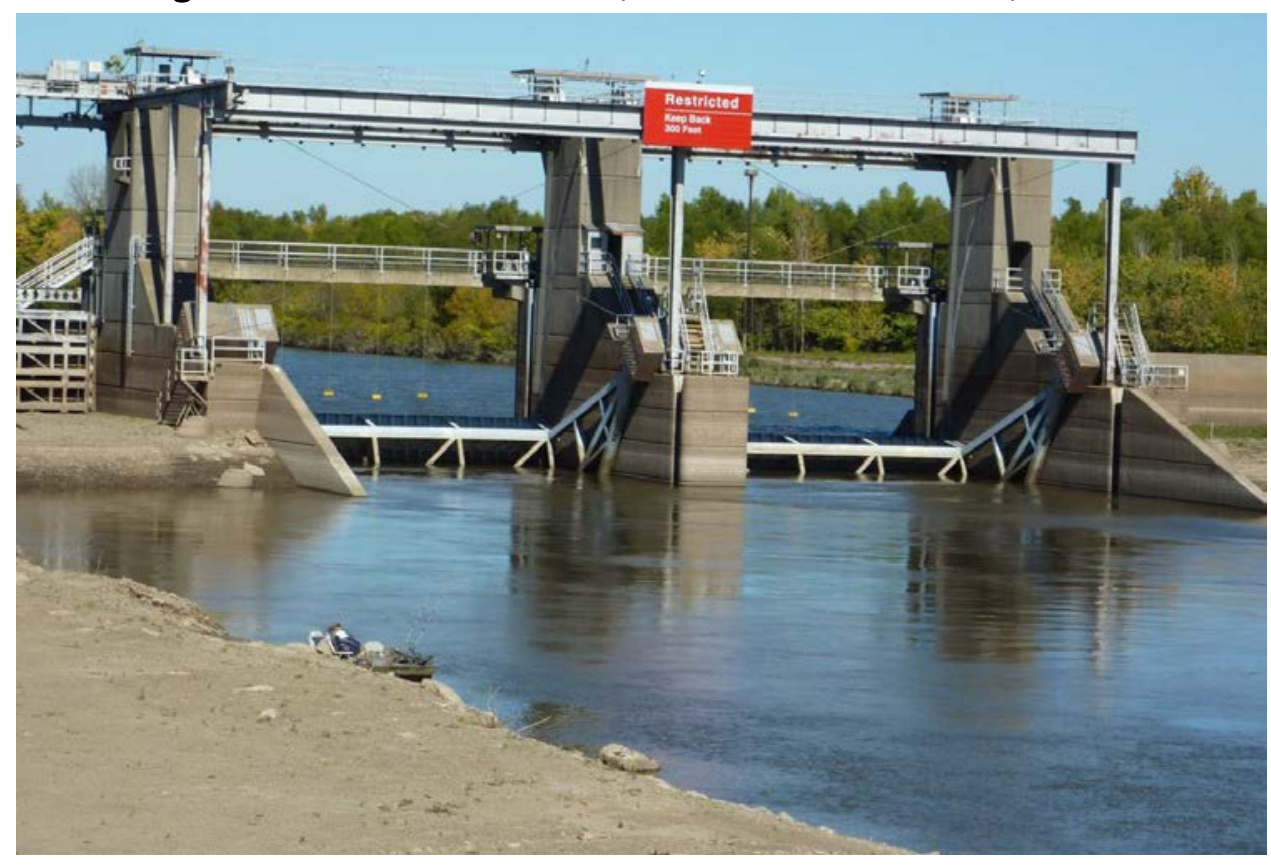

Figure 14. Rod ends at Kaskaskia Lock and Dam (left); broken rod (right).
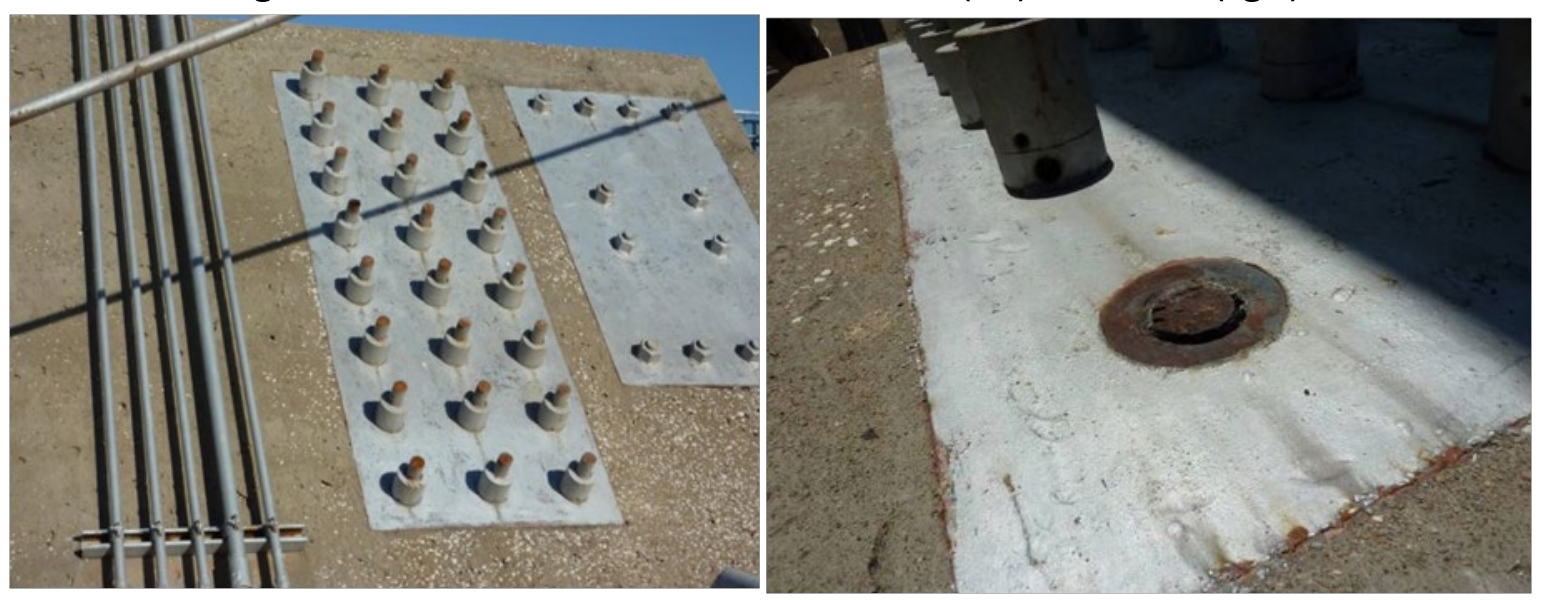

Ultrasonic data were obtained for 111 of the 112 tendon rods (rod P3W-A3 was broken). Unfortunately, the increased wave attenuation due to absorption by grout prevented shear wave measurements for all rod lengths. However, longitudinal wave measurements indicated that no rods were broken internally. All length measurements using the longitudinal wave agreed very closely with as-built lengths. We can also infer that rod tension when the grout set has remained the same. The rods are being held firmly in place by the grout, as indicated by the broken rod that remains and cannot be extracted. Appendix C includes the longitudinal velocities for all rods measured. The average longitudinal velocity was $239,755 \mathrm{in} / \mathrm{s}$ and the standard deviation was $772 \mathrm{in} / \mathrm{s}$, excluding the broken rod. 
There was also a large variation of longitudinal wave attenuation among the rods. For example, a longitudinal measurement was obtained from rod P1E-D3 using a 2.25 MHz sensor driven at $400 \mathrm{~V}$, which had 24\% echo height using $110 \mathrm{~dB}$ of receiver gain. On another rod (P1E-D2, which was right next to P1E-D3), the same sensor, again driven at $400 \mathrm{~V}$, gave an $88 \%$ echo height using $96 \mathrm{~dB}$ of receiver gain (Figure 15).

Figures 15 and 16 each show both the simplicity and difficulty of making field measurements. These figures contain screen captures from the ultrasonic detector instruments during data collection at the Illinois location.

The screen capture and table on the left in both Figures 15 and 16 were produced by a normal echo from a "normally" behaving 50-ft rod (P1E-D2) using a 0.75-in. diameter 2.25 MHz sensor. (This sensor was used for all longitudinal mode testing.) Receiver gain is $96 \mathrm{~dB}$. Note that the height of the echo is $88 \%$ of full screen height and the bottom axis is not noisy. The red line is the gate, ${ }^{*}$ with a level of $30 \%$ of screen height. The distance the wave traveled to the end of the rod is $618.400 \mathrm{in}$. (51.5 ft).

Figure 15. “Normal” rod with $2.25 \mathrm{MHz}$ sensor (left); “abnormal” rod with $2.25 \mathrm{MHz}$ sensor (right).

\begin{tabular}{|c|c|c|c|c|c|c|c|c|c|}
\hline & & & & & & & & & \\
\hline & & & & & & & & & \\
\hline & & & & & & & & & \\
\hline & & & & & & & & & \\
\hline & & & & & & & & & \\
\hline & & & & & & & & & \\
\hline & & & & & & & & & \\
\hline & & & & & & & & & \\
\hline & & & & & & & & & \\
\hline 300.00 & $\begin{array}{r}0 \\
\mathrm{D}: 61\end{array}$ & $\begin{array}{l}\text { S DAM } \\
400 \mathrm{in}\end{array}$ & in) & 700.00 & 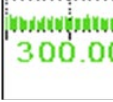 & 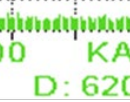 & $\begin{array}{l}\text { wownownow } \\
\text { S DAM } \\
\text {.000in }\end{array}$ & $\begin{array}{l}\text { (in) } \\
\text { (in) } 24 \%\end{array}$ & $\begin{array}{l}\text { Woustophawily } \\
700.00\end{array}$ \\
\hline CAL & Range & Delay & Vel & Zero & CAL & Range & Delay & Vel & Zero \\
\hline & 400.00 & 300.000 & 0.2307 & 0.000 & & 400.00 & 300.000 & 0.2307 & 0.000 \\
\hline & Gain & Ref & & & & Gain & Ref & & \\
\hline & 96.0 & 82.5 & & & & 110.0 & 82.5 & & \\
\hline AMP & Freq & Detect & Reject & Contour & AMP & Freq & Detect & Reject & Contour \\
\hline & $2.0 \mathrm{MHZ}$ & Full & 0 & 0 & & $2.0 \mathrm{MHZ}$ & Full & 0 & 0 \\
\hline TX & Tx Width & Damping & Volts & PRF MAX & TX & Tx Width & Damping & Volts & PRF MAX \\
\hline Single & 360 & $400 \mathrm{Ohms}$ & $400 \mathrm{v}$ & $35 \mathrm{~Hz}$ & Single & 250 & $400 \mathrm{Ohms}$ & $400 \mathrm{v}$ & $35 \mathrm{~Hz}$ \\
\hline GATE 1 & State & Start & Width & Level & GATE 1 & State & Start & Width & Level \\
\hline & $O n+V e$ & 466.400 & 384.000 & 30.0 & & $\mathrm{On}+\mathrm{Ve}$ & 466.400 & 384.000 & 20.0 \\
\hline
\end{tabular}

\footnotetext{
* The value of the peak must be greater than the gate level and within the distance interval for the instrument to display the distance (horizontal axis) and the amplitude (vertical axis) values of the peak.
} 
Figure 16. “Normal” rod with $2.25 \mathrm{MHz}$ sensor (left); “normal” rod with 1.0 MHz sensor (right).

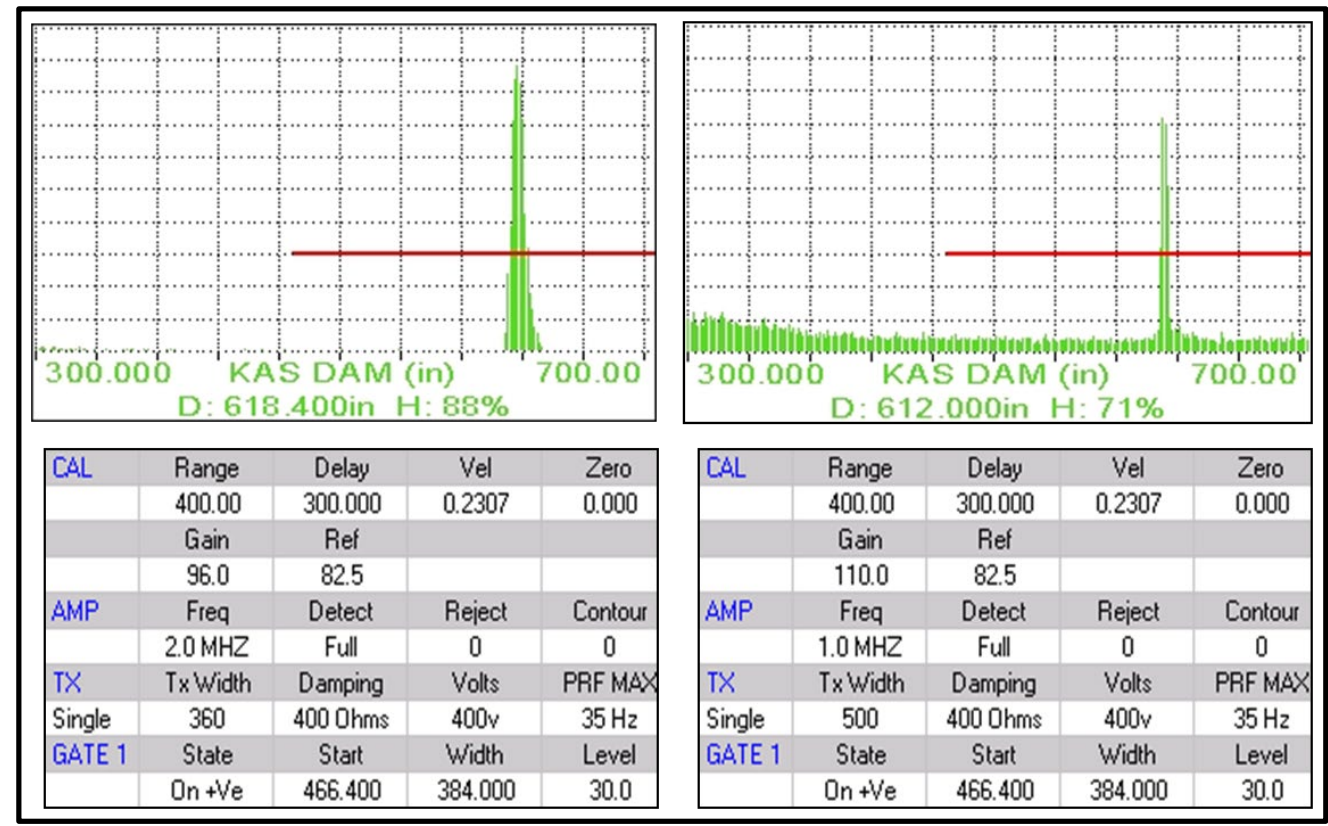

An echo from an "abnormally" responding 50-ft rod (P1E-D3) using the standard $0.75 \mathrm{in}$. diameter 2.25-MHz sensor with a higher gain of $110 \mathrm{~dB}$ is shown on the right in Figure 15. The change in decibels from the normal situation is $-25.3 \mathrm{~dB}$, or just $5.4 \%$ the strength of the normal signal from the standard sensor on a normal rod. There is noise along the bottom axis due to the high gain. The red line is the gate level, set to $20 \%$ of screen height. The level was reduced to measure the lower echo height. The specific reason for the high ultrasonic wave attenuation in this rod is not known. However, it may indicate that a larger surface area of the rod, compared to the other rods, is in direct contact with the grout. Snell's Law predicts that a large percentage of the wave $(63.4 \%$ for concrete, which has a base composition similar to grout) is refracted into the grout and absorbed due to the grout's higher attenuation coefficient. The more of the rod's surface that is in direct contact with the grout, the more the waves are refracted into the grout and absorbed, leading to the appearance of increased wave attenuation in the rod. Therefore, the attenuation in the rod is most probably directly related to the amount of grout in direct contact with the rod. The increased attenuation of the waves is another indication that the rods are firmly held in the grout.

The screen capture and table on the right of Figure 16 are from the "normally" behaving 50-ft long rod (P1E-D2) using a 1.0 in. diameter 1.0-MHz sensor. Note that a much higher gain (110 dB) compared to the $2.25-\mathrm{MHz}$ 
sensor was required to obtain an acceptable signal; the height of the echo is $71 \%$ of full screen height; and there is noise along the bottom axis. The gate is still set at a level of $30 \%$ of screen height. The change in decibels of the peak from the normal situation is $-15.9 \mathrm{~dB}$. ${ }^{*}$ This means the peak signal strength using the $1.0 \mathrm{MHz}$ sensor is $0.16^{\dagger}(16 \%)$, the peak signal strength of the $2.25 \mathrm{MHz}$ sensor. Ultrasonic theory says that attenuation decreases with decreasing frequency. It is unknown why, on this rod, a lower-frequency signal is attenuated more than a higher-frequency signal.

There are many possible difficulties with ultrasonically measuring the tension of anchor rods in situ. The greatest is the attenuation of the acoustic signals, especially that of the shear wave. Additional wave propagation problems are reflection, refraction, beam spread, and coupling of the signal to the rod from the transducer. Unfortunately, these difficulties result entirely due to the physics of the problem and are very difficult to overcome. The measurement will also be dependent on the medium surrounding the rod, e.g., air, grease, or grout.

To compute the tension in the rods, according to Equations 14 and 15, the longitudinal or shear velocity must be known in the unstressed state and in the stressed state. In the field, these measurements are difficult to perform because, to compute the acoustic velocity, the actual length of the rod must be divided by the time of flight measured by the flaw detector. In the field, the length of the rod cannot be measured to the precision required to calculate the unknown value of the tension. An alternative approach to the measurement of tension is presented below.

Equations 14 and 15 can be rewritten in terms of the time of flight. This approach removes the length dependence of the rods, which is reasonable because the length of the rods does not change over time except for temperature effects, which are minor variabilities. The new equations for tension become:

$$
d \sigma=-\frac{E}{L_{l}}\left(\frac{d t_{l}}{t_{l 0}}\right)
$$

* $-15.9=\left[96-\left(110+20 \times \log _{10}(88 / 71)\right)\right]$

$+0.16=\left[10^{-15.9 / 20}\right]$ 


$$
d \sigma=-\frac{E}{L_{s}}\left(\frac{d t_{s}}{t_{s 0}}\right)
$$

where, $\mathrm{dt}_{\mathrm{l}}$ and $\mathrm{dt}_{\mathrm{s}}$ are the change in longitudinal and shear transit time, respectively and $t_{10}$ and $t_{s 0}$ are the longitudinal and shear transit time, respectively, in the unstressed state.

As the rods are installed and tensioned, the value of the stress is measured to ensure tensioning to the appropriate value (typically near the yield stress). After the rods are installed and tensioned, the ends of the rods are typically cut for appropriate sizing. For better signal transmission, especially for longer rods, both ends of the rod should be smoothed. Once in this final state, the longitudinal and shear time of flights should be measured at the known value of tension. Given that the initial post-tensioned rod stress and longitudinal or shear time of flights are known, the corresponding time of flight in the unstressed state can be calculated from Equations 21 and 22 according to:

$$
\begin{aligned}
& t_{l 0}=\frac{t_{l, p o s t}}{\left(1-\sigma_{\text {post }} \frac{L_{L}}{E}\right)} \\
& t_{s 0}=\frac{t_{s, \text { post }}}{\left(1-\sigma_{\text {post }} L_{s}\right)}
\end{aligned}
$$

where $t_{1, p o s t}$ and $t_{s, p o s t}$ are the time of flights at the post-tensioned stress level $\sigma_{\text {post }}$ for longitudinal and shear waves, respectively.

Over time, the rods will undergo degradation and the stress will change, which will result in a change in the time of flight for the longitudinal and shear waves. By measuring the longitudinal and shear time of flights and combining Equations 23 and 24 with Equations 21 and 22, the prediction of the stress in the rods can be found using the following equations:

$$
\begin{aligned}
& \sigma\left(t_{l}\right)=\frac{E}{L_{l}}\left[1-\frac{t_{l}}{t_{l, p o s t}}\left(1-\sigma_{\text {post }} \frac{L_{l}}{E}\right)\right] \\
& \sigma\left(t_{s}\right)=\frac{E}{L_{s}}\left[1-\frac{t_{s}}{t_{s, p o s t}}\left(1-\sigma_{\text {post }} \frac{L_{s}}{E}\right)\right]
\end{aligned}
$$

where $\sigma\left(\mathrm{t}_{\mathrm{l}}\right)$ is the stress in the rod as a function of the time of flight for the longitudinal wave ( $\left.\mathrm{t}_{\mathrm{l}}\right)$ and $\sigma\left(\mathrm{t}_{\mathrm{s}}\right)$ is the stress in the rod as a function of the time of flight for the shear wave $\left(t_{s}\right)$. 
It is recommended that Equation 25 be used to predict stress and that Equation 26 be used as an alternative when it is not possible to use Equation 25. This is because the stress shows a stronger dependence on the longitudinal time of flight versus shear time of flight. Thus, to predict the stress in a post-tensioned rod, Equation 25 can be used in conjunction with knowledge of the initial post-tensioned stress, initial longitudinal time of flight, present longitudinal time of flight, and the material parameters $\mathrm{E}$ and $\mathrm{L}$.

If the material parameter $\mathrm{L}_{1}$ is unknown, it must be measured in a laboratory setting. This can be done by using a rod that is identical in material to the anchoring rods used in the field. The rod should be placed in a load frame and the strain and ultrasonic longitudinal velocity should be measured as a function of stress. The same frequency transducer should be used (and preferably the same equipment) as in the field measurements. The longitudinal velocity should be normalized to its unstressed value and then plotted versus the measured strain. The data should be fit to a straight line and the slope should be identified with the parameter $\mathrm{Ll}$ according to Equation 12. 


\section{Conclusions and Future Work}

\subsection{Conclusions}

This work developed a theory and test technique for quantitatively determining tension in concrete-embedded structural steel members. A theoretical basis was established for bulk tension measurements and an acoustic propagation model was developed and implemented using commercially available software. The theory and model were verified in the laboratory using simple steel rods as test specimens. The technology and method of application were awarded a U.S. patent (McInerney et al. 2009).

Field tests of a new ultrasonic tension measurement method were conducted at three Corps of Engineers dams, located in Oklahoma, Georgia, and Illinois.

At Keystone Dam in Oklahoma, we demonstrated that the technique could measure longitudinal and shear velocity in rods up to $50 \mathrm{ft}$ long. For unknown reasons, the longitudinal wave propagated through only six of the 20 rods, and the shear wave propagated through only one rod. In retrospect, based on the results of the West Point tests, we were probably very near the measurement limit of our system.

At West Point Dam in Georgia, we obtained measurements on each of the 37 rods tested, but the quality and consistency of the signal varied. This led to wide variability in the measured longitudinal and shear velocities leading to the conclusion that the tension in the rods varied greatly. One would expect the rods to change tension uniformly and without such an extreme variance. We were able to obtain longitudinal and shear wave measurements on all 37 rods, although obtaining a shear measurement was difficult for a few of the longer ( $48 \mathrm{ft}$ ) rods. This result led us to conclude that our current instrumentation and transducers are suitable for use on rods up to $45 \mathrm{ft}$ in length.

At Kaskaskia Lock and Dam in Illinois, ultrasonic data were obtained for each of the 111 rods tested. There was a large variation of longitudinal wave attenuation among the rods. Unfortunately, as noted in the main text, the grout in the tendon conduit prevented shear wave measurements for all rods. However, because all length measurements using the longitudinal wave agreed very closely with as-built lengths, we can conclude that 
none of the 111 rods is broken. We can also infer that the tension that was in the rods when the grout set has remained the same.

While the tension prediction theory was confirmed in the laboratory tests, the tension could not be calculated for any of the field sites. This is because we were not able to obtain the longitudinal or shear velocities in an unstressed state. Precise measurements of the longitudinal and shear velocities were also not possible due to the lack of knowledge of the precise length of the rods in the tensioned state, which is necessary to accurately compute longitudinal and shear velocity. The alternative approach summarized in Equations 25 and 26 also could not be used since the unstressed time of flights were not available. In the future, it is recommended that both the post-tensioned stress and longitudinal time of flight be measured at installation so that future tension values can be computed using Equations 25 and 26 with the additional information provided by the measured current longitudinal time of flight.

When perfected, the benefits of this acoustic NDE tension measurement technology will include:

- rapid, noninvasive tension measurement of embedded steel rods in the field

- ease of measurement where at least one rod end is available, even where access is difficult

- cost reduction by a factor of 10 compared with the present lift-off testing method

- facilitation of more frequent testing and improved structural evaluation.

\subsection{Future work}

There are several challenges with perfecting this tension measurement method for use in the field, mostly involving the propagation of the ultrasonic waves and precise determination of rod material and ultrasonic parameters. This technology is potentially suitable for future field work where the acoustic velocities, material parameters, and material dimensions can be accurately measured upon installation and post-tensioning of the rods.

An error analysis should be performed on the measurement and computation methods; specifically, of how instrument quantization and reading errors, and possible errors in material property values, affect the tension 
computation. This analysis should also include the required accuracy of the measurements and the instruments and conditions required to obtain that accuracy.

Further laboratory studies of wave propagation may be done using the ERDC Anchor Rod Test Bed, located at the Engineer Research and Development Center, Coastal and Hydraulics Laboratory, Vicksburg, MS. This facility accommodates the testing of $70 \mathrm{ft}$ long rods under tension and with several different conduit-filler materials. 


\section{Bibliography}

Auld, B. A. 1990. Acoustic Fields and Waves in Solids, Vol I \& II, 2 d edition. Malabar, FL: Krieger Publishing Company.

Bray, D. E. 2000. Current Directions of Ultrasonic Stress Measurement Techniques, Roma 2000; 15th WCNDT. http://www.ndt.net/article/wcndt00/papers/idn647/idn647.htm.

Bray, D. and R. K. Stanley. 1997. Nondestructive Evaluation. Boca Raton, FL: CRC Press.

Ensminger, D. 1973. Ultrasonics. New York: Marcel Dekker, Inc.

Filipczynski, L., Z. Pawlowski, and J . Wehr. 1966. Ultrasonic Methods of Testing Materials. London: Butterworth \& Co.

HQUSACE (Headquarters, U.S. Army Corps of Engineers). 2000. Design of Spillway Tainter Gates. Engineer Manual (EM) 1110-2-2702. Washington, DC: HQUSACE.

Krautkramer, J osef, and Herbert Krautkramer. 1990. Ultrasonic Testing of Materials, 4th ed. Springer-Verlag.

McInerney, Michael K., Sean W. Morefield, Vincent F. Hock, Victor H. Kelly, and John M. Carlyle. 2009. Device for Measuring Bulk Stress via Insonification and Method of Use Therefor. U.S. Patent Number 7,614,303 B2.

Murnaghan, F. 1937. Finite Deformations of an Elastic Solid. American J ournal of Mathematics. 


\section{Appendix A: Corrosion Prediction Model}

The Imagine3D software was also used to simulate the addition of a small corrosion pit to the 19-in. long by 1.25-in. diameter steel rod that we used in the previous model. We simulated a corrosion pit by removing an ellipsoid of material from the top of the steel rod. (Note that this is the same as assuming that the ellipsoid is filled with air.) The ellipsoid extended $0.1 \mathrm{in}$. into the rod, had a diameter across the ultrasonic beam of $0.1 \mathrm{in}$., and had a diameter along the ultrasonic beam of $0.25 \mathrm{in}$.

The top plot in Figure A-1 shows the reflections obtained from the ellipsoid. The green rays coming off of the ellipsoid are longitudinal waves, while the purple rays coming off of the ellipsoid are shear waves. It can be seen that no ray is heading directly back to the sensor - both the longitudinal waves and the shear waves are heading for the bottom surface of the rod, where they will reflect (and mode convert) before they arrive back at the sensor. This increased travel distance is what accounts for the first echo from the ellipsoid being shown at the 10-in. distance mark on the middle plot of Figure A-1, instead of the expected 9 in.

The fact that there are both longitudinal and shear waves reflecting off the ellipsoid suggests that there will be more than one echo arriving at the sensor from the ellipsoid. Indeed, this is true; the middle plot of Figure A-1 shows that there is another echo at about the 11-in. distance mark, and additionally there are echoes shown at the 14-, 15-, 16-, 17-, and 18-in. distances as well. For reference, the bottom plot of Figure A- 1 shows what echoes would be expected using the same gain level for a 19-in. long by 1.25-in. diameter steel rod with no corrosion. 
Figure A-1. (Top) Simulation of corrosion pit in 19-in. long by 1.25-in. diameter steel rod. The corrosion pit is located on top of the rod at a horizontal distance of 9 in. from the sensor. (Middle) Echoes created by the corrosion pit. (Bottom) Echoes obtained from a steel rod with no corrosion.

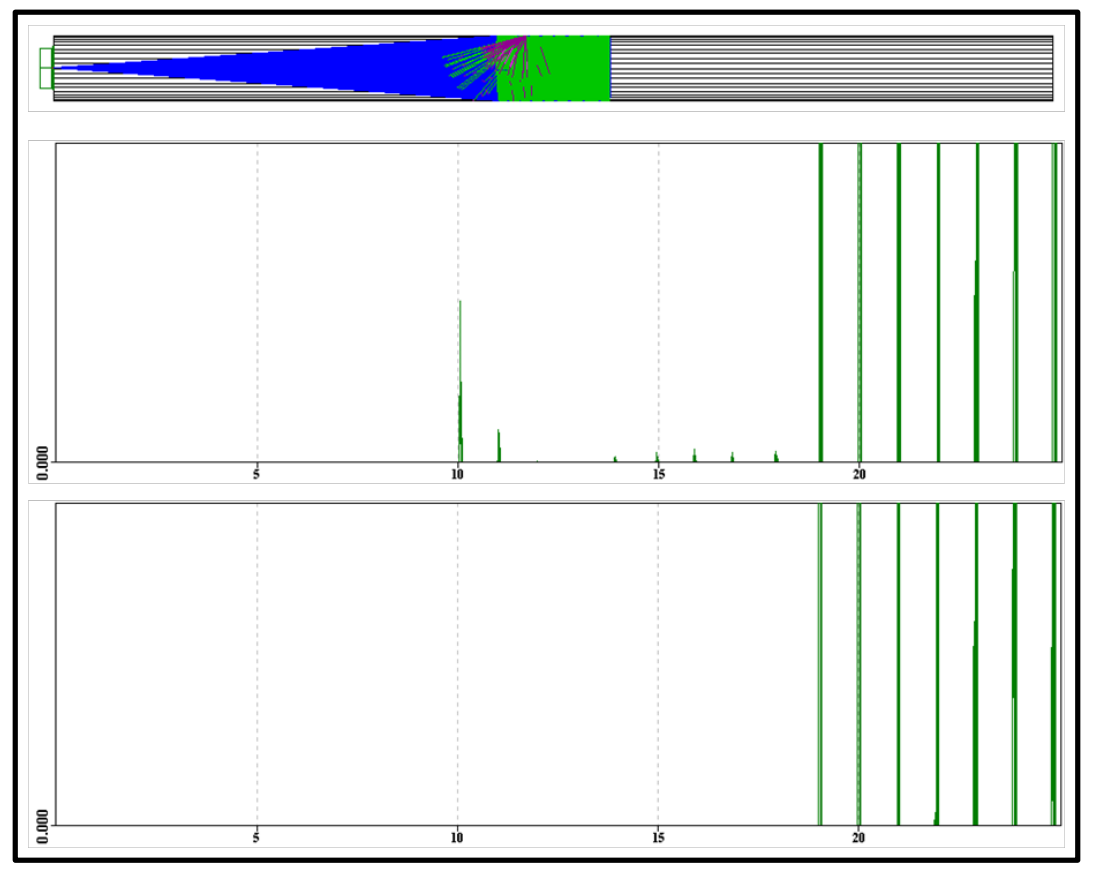

The corrosion prediction model that we have developed is composed of Equations A-1, A-2, and A-3 and the accurate ultrasonic reflector modeling capabilities of Imagine3D. Equation A-1 provides us with the ability to calculate the reflection coefficient, $\alpha_{r}$, which predicts how much energy will reflect from a patch of corrosion:

$$
\alpha_{r}=\left\{\left(\rho_{2} V_{2}-\rho_{1} V_{1}\right) /\left(\rho_{2} V_{2}+\rho_{1} V_{1}\right)\right\}^{2}=\left\{\left(Z_{2}-Z_{1}\right) /\left(Z_{2}+Z_{1}\right)\right\}^{2}
$$

where:

$$
\begin{aligned}
& \rho_{1}=\text { the density of medium } 1 \\
& \rho_{2}=\text { the density of medium } 2 \\
& \mathrm{~V}_{1}=\text { the speed in medium } 1 \\
& \mathrm{~V}_{2}=\text { the speed in medium } 2 \\
& \mathrm{Z}_{1}=\text { the acoustic impedance of medium } 1 \\
& \mathrm{Z}_{2}=\text { the acoustic impedance of medium } 2 .
\end{aligned}
$$

Equation A-2 is Snell's Law, and gives us the ability to determine the angle at which a reflected ultrasonic wave will travel, given the speed of sound of the wave mode in the medium: 


$$
\sin \alpha / V_{11}=\sin \beta / V_{s 1}=\sin \gamma / V_{12}=\sin \delta / V_{s 2}
$$

where:

$$
\begin{aligned}
\mathrm{V}_{11}= & \text { the speed of the longitudinal wave in medium } 1 \\
\mathrm{~V}_{\mathrm{s} 1}= & \text { the speed of the shear wave in medium } 1 \\
\mathrm{~V}_{\mathrm{l} 2}= & \text { the speed of the longitudinal wave in medium } 2 \\
\mathrm{~V}_{\mathrm{s} 2}= & \text { the speed of the shear wave in medium } 2 \\
\alpha= & \text { the angle from a perpendicular to the boundary surface to the } \\
& \text { propagation direction of the longitudinal wave in medium } 1 \\
\beta= & \text { the angle from a perpendicular to the boundary surface to the } \\
& \text { propagation direction of the shear wave in medium } 1
\end{aligned}
$$

When we are working with long rods, we need to know how the ultrasonic beam behaves far from the sensor. Equation A-3 allows us to calculate the divergence angle $\theta$, of an ultrasonic beam in the far field:

$$
\theta=\arcsin (\mathrm{k} \lambda / 2 \mathrm{a})
$$

where:

$$
\begin{aligned}
& \mathrm{a}=\text { the radius of the sensor face } \\
& \mathrm{k}=\text { a constant with a value of } 0.5 \text { or } 0.6 \\
& \lambda=\text { the wavelength of the ultrasound. }
\end{aligned}
$$

Equations A-1, A-2, and A-3 are built into Imagine3D. This simulation program thus provides a very convenient method for modeling the effect that corrosion will have on ultrasonic signals propagating in any medium of any size and shape.

Figure A-1 shows the result of one such model being carried out. This figure clearly shows that detecting corrosion in a rod is not an easy task. Here a single small corrosion pit generated seven different ultrasonic echoes, none of which are at the expected distance of 9 in. Furthermore, five of the echoes created by the corrosion pit are located over 4 in. away from the main ultrasonic echo from the pit, and they appear to get larger the further 
away they get from the pit. However, the complexity of Figure A- 1 is precisely the reason why the modeling of corrosion via Imagine3D is potentially such a valuable tool.

Without modeling the typical development approach that would be followed for using ultrasound to find corrosion in buried steel rods would be to manufacture full size test specimens. Each of these test specimens would contain a single corrosion patch at a single distance, and a specific technique would be developed via trial and error using many different sensors to find this one patch of corrosion.

To obtain an effective inspection of the complete rod where corrosion could occur anywhere in any amount would require dozens of these rods during the inspection development in a process that would clearly be very expensive and time consuming.

With modeling, however, the development process changes drastically. Using drafting software such as AutoCAD, different types of corrosion can be modeled electronically. For example, corrosion patches could contain pits, they could contain cracks, they might be layered (as if they were experiencing exfoliation), they could be empty, they might contain water and iron oxide, etc. Each of these simulated patches can then be inserted electronically into a scale model of the rod (and the rod model could contain threads, wedge nuts, welds, etc.) at any desired distance, angular placement or orientation. Sensors of different frequencies and diameters could then be used to illuminate the corrosion, and the ultrasonic beams could be modified via the use of wedges and ultrasonic lenses to have any number of interesting and useful characteristics. The best possible inspection techniques can be quickly developed from these electronic experiments; the modeling could then be confidence-checked by manufacturing only one or two full size specimens. The big benefit of the modeling process is that it is much more time- and cost-effective than the traditional inspection development method described in the preceding paragraph.

Before we reach this promising future, however, it will be necessary to test Imagine3D more thoroughly than was possible during the present development task. This work checked the accuracy of the Imagine3D modeling of echoes produced in a steel rod by actually doing the experiment using a real ultrasonic instrument, a real sensor, and a real rod (Figure 3, p 8). 
Similarly, it will be necessary to do the same experiment with corrosion located somewhere along the surface of the rod. In other words, we will have to machine the ellipsoid used in Figure A-1 and use a real ultrasonic instrument, a real sensor, and a real rod to see if we obtain the echoes that Image3D produced in Figure A-1. 


\section{Appendix B: Laboratory Experiments with Other Rods}

B-1.Plots of the scaled longitudinal velocity as a function of strain for independent trial runs with various types of steel rods. Each set of data is fit to a linear curve and the average slope, given by $<m>$ is recorded.
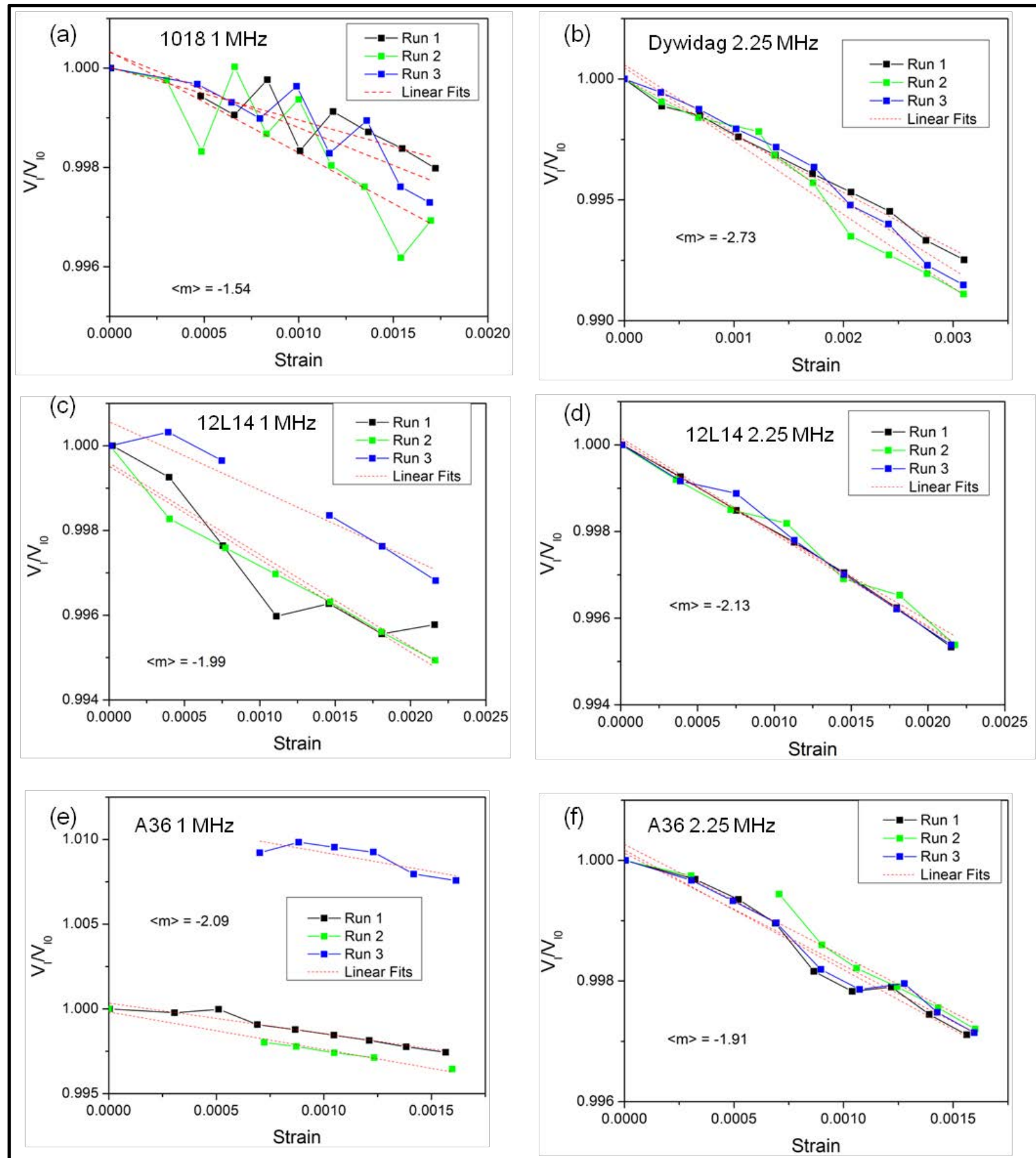
Figure B-2. Plots of the scaled shear velocity as a function of strain for independent trial runs with the same types of steel rods as is presented in Fig. B-1. Each set of data is fit to a linear curve and the average slope, given by $<m>$ is recorded.
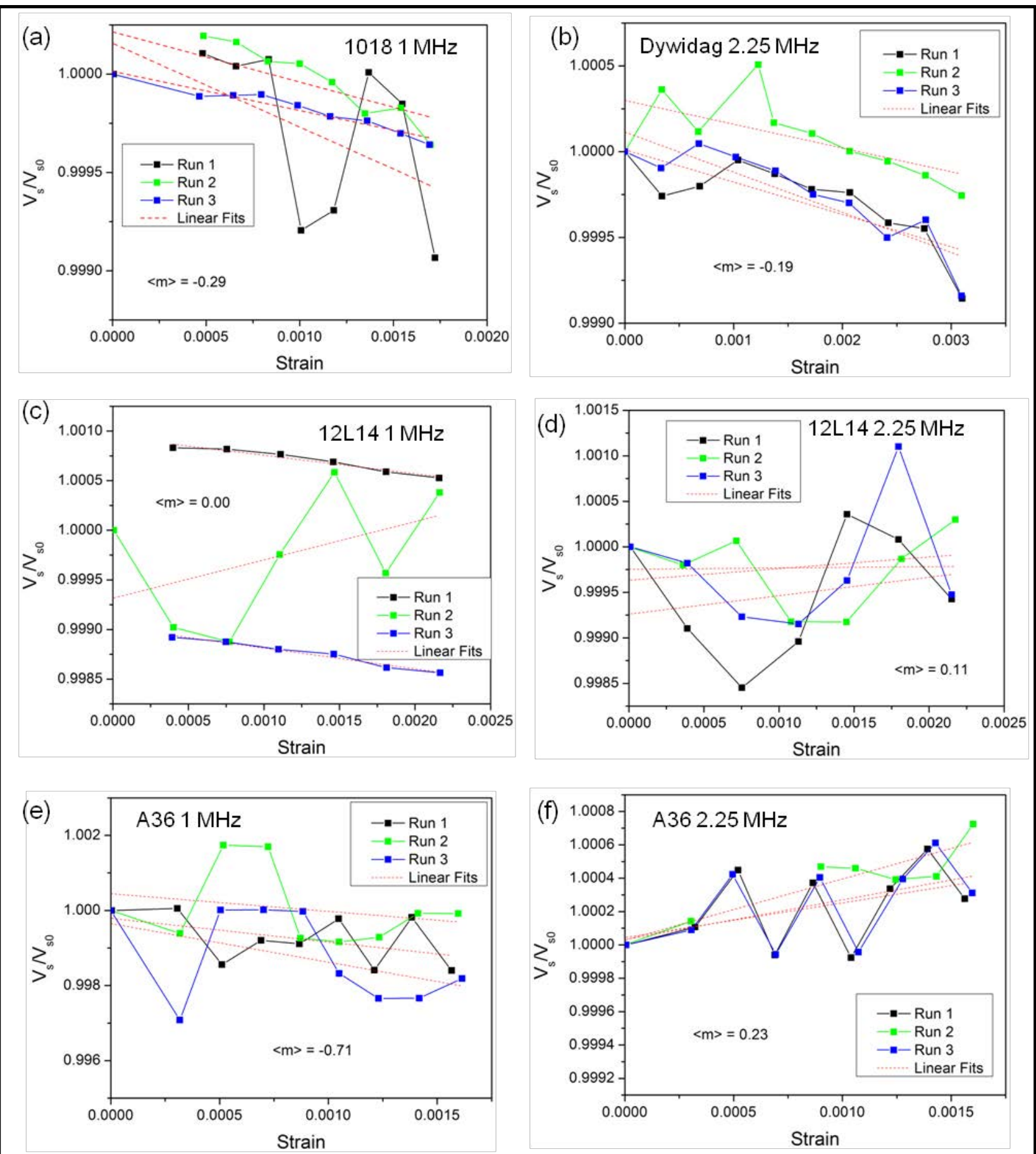


\section{Appendix C: Kaskaskia Lock and Dam data}

Table C-1. Longitudinal velocity of rods at the Kaskaskia Lock and Dam.

\begin{tabular}{|c|c|c|c|c|c|c|c|}
\hline Rod & $V_{1}($ in $/ s)$ & Rod & $V_{I}($ in/s) & Rod & $V_{1}($ in $/ s)$ & Rod & $V_{1}($ in/s) \\
\hline Rod No. P1W-A1 & 239423 & Rod No. P2W-A1 & 239423 & Rod No. P2E-A2 & 238982 & Rod No. P3W-A3 & Broken \\
\hline Rod No. P1W-B1 & 239155 & Rod No. P2W-B1 & 240767 & Rod No. P2E-B2 & 240767 & Rod No. P3W-B3 & 240095 \\
\hline Rod No. P1W-C1 & 239423 & Rod No. P2W-C1 & 240159 & Rod No. P2E-C2 & 240894 & Rod No. P3W-C3 & 240894 \\
\hline Rod No. P1W-D1 & 238558 & Rod No. P2W-D1 & 240659 & Rod No. P2E-D2 & 239423 & Rod No. P3W-D3 & 238805 \\
\hline Rod No. P1W-E1 & 240179 & Rod No. P2W-E1 & 240095 & Rod No. P2E-E2 & 240767 & Rod No. P3W-E3 & 239423 \\
\hline Rod No. P1W-F1 & 238929 & Rod No. P2W-F1 & 238805 & Rod No. P2E-F2 & 239423 & Rod No. P3W-F3 & 238805 \\
\hline Rod No. P1W-G1 & 240894 & Rod No. P2W-G1 & 240894 & Rod No. P2E-G2 & 240159 & Rod No. P3W-G3 & 240159 \\
\hline Rod No. P1W-H1 & 240767 & Rod No. P2W-H1 & 240095 & Rod No. P2E-H2 & 238080 & Rod No. P3W-H3 & 238752 \\
\hline Rod No. P1E-A1 & 239423 & Rod No. P2W-A2 & 239423 & Rod No. P2E-A3 & 240159 & Rod No. P3E-A1 & 240894 \\
\hline Rod No. P1E-B1 & 238214 & Rod No. P2W-B2 & 240095 & Rod No. P2E-B3 & 239423 & Rod No. P3E-B1 & 240095 \\
\hline Rod No. P1E-C1 & 240894 & Rod No. P2W-C2 & 240894 & Rod No. P2E-C3 & 240159 & Rod No. P3E-C1 & 239423 \\
\hline Rod No. P1E-D1 & 238805 & Rod No. P2W-D2 & 238805 & Rod No. P2E-D3 & 238805 & Rod No. P3E-D1 & 240041 \\
\hline Rod No. P1E-E1 & 238617 & Rod No. P2W-E2 & 240095 & Rod No. P2E-E3 & 240095 & Rod No. P3E-E1 & 240095 \\
\hline Rod No. P1E-F1 & 238805 & Rod No. P2W-F2 & 239423 & Rod No. P2E-F3 & 239423 & Rod No. P3E-F1 & 239547 \\
\hline Rod No. P1E-G1 & 239423 & Rod No. P2W-G2 & 240159 & Rod No. P2E-G3 & 240159 & Rod No. P3E-G1 & 242071 \\
\hline Rod No. P1E-H1 & 239423 & Rod No. P2W-H2 & 240095 & Rod No. P2E-H3 & 240095 & Rod No. P3E-H1 & 239423 \\
\hline Rod No. P1E-A2 & 240894 & Rod No. P2W-A3 & 239423 & Rod No. P3W-A1 & 241189 & & \\
\hline Rod No. P1E-B2 & 238752 & Rod No. P2W-B3 & 239423 & Rod No. P3W-B1 & 239423 & & \\
\hline Rod No. P1E-C2 & 240159 & Rod No. P2W-C3 & 240159 & Rod No. P3W-C1 & 240159 & & \\
\hline Rod No. P1E-D2 & 238805 & Rod No. P2W-D3 & 238805 & Rod No. P3W-D1 & 238558 & & \\
\hline Rod No. P1E-E2 & 238349 & Rod No. P2W-E3 & 240095 & Rod No. P3W-E1 & 238752 & & \\
\hline Rod No. P1E-F2 & 238805 & Rod No. P2W-F3 & 239423 & Rod No. P3W-F1 & 238805 & & \\
\hline Rod No. P1E-G2 & 239423 & Rod No. P2W-G3 & 240159 & Rod No. P3W-G1 & 240894 & & \\
\hline Rod No. P1E-H2 & 239423 & Rod No. P2W-H3 & 240095 & Rod No. P3W-H1 & 239423 & & \\
\hline Rod No. P1E-A3 & 240159 & Rod No. P2E-A1 & 240894 & Rod No. P3W-A2 & 240894 & & \\
\hline Rod No. P1E-B3 & 239423 & Rod No. P2E-B1 & 239961 & Rod No. P3W-B2 & 240028 & & \\
\hline Rod No. P1E-C3 & 240894 & Rod No. P2E-C1 & 239423 & Rod No. P3W-C2 & 240894 & & \\
\hline Rod No. P1E-D3 & 240041 & Rod No. P2E-D1 & 239423 & Rod No. P3W-D2 & 238805 & & \\
\hline Rod No. P1E-E3 & 238886 & Rod No. P2E-E1 & 239356 & Rod No. P3W-E2 & 239692 & & \\
\hline Rod No. P1E-F3 & 239423 & Rod No. P2E-F1 & 240041 & Rod No. P3W-F2 & 239423 & & \\
\hline Rod No. P1E-G3 & 240159 & Rod No. P2E-G1 & 240527 & Rod No. P3W-G2 & 240159 & & \\
\hline Rod No. P1E-H3 & 239155 & Rod No. P2E-H1 & 240262.8 & Rod No. P3W-H2 & 239356 & & \\
\hline
\end{tabular}




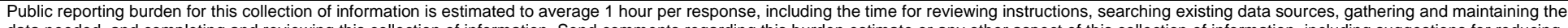

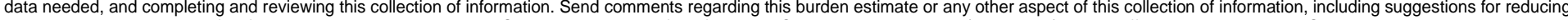

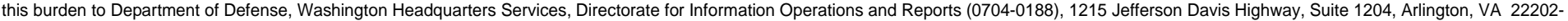

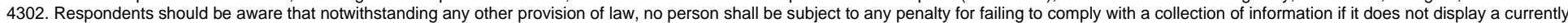
valid OMB control number. PLEASE DO NOT RETURN YOUR FORM TO THE ABOVE ADDRESS.

\begin{tabular}{l|c|c|} 
1. REPORT DATE & 2. REPORT TYPE & 3. DATES COVERED (FrOm - TO)
\end{tabular}

Final Technical Report

Acoustic Nondestructive Testing and Measurement of Tension for Steel Reinforcing

Members

5 a. CONTRACT NUMBER

5b. GRANT NUMBER

5c. PROGRAM ELEMENT

\section{AUTHOR(S)}

Michael K. McInerney, Matthew W. Brenner, Sean W. Morefield, Robert A. Weber, and John M. Carlyle

\section{5 d. PROJECT NUMBER}

611102AH68

5e. TASK NUMBER

5f. WORK UNIT NUMBER

98KJ26

8. PERFORMING ORGANIZATION REPORT NUMBER

ERDC/CERL TR-21-20

10. SPONSOR/MONITOR'S ACRONYM(S)

USACE

11. SPONSOR/MONITOR'S REPORT NUMBER(S)

\section{DISTRIBUTION I AVAILABILITY STATEMENT}

Approved for public release; distribution unlimited.

\section{SUPPLEMENTARY NOTES}

\section{ABSTRACT}

Many concrete structures contain internal post-tensioned steel structural members that are subject to fracturing and corrosion. The major problem with conventional tension measurement techniques is that they use indirect and non-quantitative methods to determine whether there has been a loss of tension. We have developed an acoustics-based technology and method for making quantitative tension measurements of an embedded, tensioned steel member. The theory and model were verified in the laboratory using a variety of steel rods as test specimens. Field tests of the method were conducted at three Corps of Engineers dams, located in Oklahoma, Georgia, and Illinois. Measurements of the longitudinal and shear velocity were able to be made on rods up to $50 \mathrm{ft}$ long. Not all rods of this length were able to be measured and the quality and consistency of the signal varied. There were fewer problems measuring the longitudinal velocity than shear velocity. While the tension predictions worked in the laboratory tests, the tension could not be accurately calculated for any of the field sites. This is because we were not able to obtain the longitudinal or shear velocities in an unstressed state or precise measurements of the longitudinal and shear velocities due to the lack of knowledge of the precise length of the rods in the tensioned state.

\section{SUBJECT TERMS}

Nondestructive testing; Reinforced concrete; Hydraulic structures; Ultrasonic waves; Steel, Structural; Tensile architecture

\section{SECURITY CLASSIFICATION OF:}$$
\text { a. REPORT }
$$$$
\text { Unclassified }
$$

\author{
b. ABSTRACT \\ Unclassified
}

\begin{tabular}{c|c|c} 
17. LIMITATION & $\begin{array}{c}\text { 18. NUMBER } \\
\text { OF ABSTRACT }\end{array}$ & 19 \\
OF PAGES & & \\
\cline { 3 - 3 } & & 50
\end{tabular}

\section{9 a. NAME OF RESPONSIBLE PERSON \\ 19b. TELEPHONE NUMBER (include area code)}

